\title{
AGGREGATION IN A MODEL OF PRICE COMPETITION
}

\section{Martin Peitz}

WP-AD 99-26

Correspondence to: University of Alicante. Departamento de Fundamentos del Análisis Económcio. Ctra. San Vte. del Raspeig, s/n. 03071 Alicante, Spain. Tel: 965903563 Ext: 3218. E-Mail: peitz@merlin.fae.ua.es

Editor: Instituto Valenciano de Investigaciones Económicas, s.a.

First Edition Diciembre 1999

Depósito Legal: V-5426-1999

IVIE working papers offer in advance the results of economic research under way in order to encourage a discussion process before sending them to scientific journals for their final publication.

* This paper replaces my paper with the title "Aggregation and Strategic Complementarity in a Model of Price Competition" (Peitz, 1995). I would like to thank Egbert Dierker, Jean-Michel Grandmont and Birgit Grodol for helpful discussions. Financial support by the Deutsche Forschungsgemeinschaft - Sonderforschungsbereich 303 and the Instituto Valenciano de Investigaciones Económicas (IVIE) is gratefully acknowledged. 


\section{AGGREGATION IN A MODEL OF PRICE COMPETITION}

\section{Martin Peitz}

WP-AD 99-26

\section{A B S T R A C T}

In a model of price competition single-product firms compete for consumers. Consumers purchase a variable quantity of one of the differentiated goods. The paper provides results on equilibrium existence when consumers are heterogeneous in their evaluation of the differentiated goods among each other, their evaluation of the differentiated goods relative to the outside good, and heterogeneous in income. Furthermore, I provide sufficient conditions for dominance solvability and monotone comparative statics.

KEYWORDS: Price Competition; Imperfect Competition; Heterogeneous Demand; Oligopoly Theory; Product Differentiation. 


\section{Introduction}

Consider a differentiated oligopoly with a finite number of one-product firms competing in prices. It is known since the work of Roberts and Sonnenschein (1977) that there are robust non-existence results of Nash equilibrium in pure strategies. Suppose that there exists a market with two types of consumers characterized by their individual demand function. Even if equilibrium existence can be shown in any market with a single type of consumers non-existence might result in the presence of two types. The problem of non-existence of equilibrium is due to a lack of restrictions derived for market, i.e. aggregate, demand. The lack of restrictions on market demand can be seen as the most fundamental problem in establishing a general theory of price competition in partial equilibrium. A foundation of imperfect competition then consists of the analysis of models which go beyond a representative consumer specification or particular examples with heterogeneous consumers and which address and partially resolve the problem. Given the importance of imperfect competition in fields such as industrial organization, international trade, economic geography, and regional science such models and the insights they provide might be of interest to a wide audience.

In order to establish existence of equilibrium previous work has concentrated on models in which the best response correspondences of the firms are convex-valued. In the framework of discrete choice with unit demand or unit elastic demand, positive results have been obtained by Caplin and Nalebuff (1991b), Dierker (1991), and Peitz (1997). The theme of their and my paper is that distributional assumptions can generate strong regularities of aggregate demand. The main contribution of this paper is to avoid functional form assumptions on individual demand and to show the existence of equilibrium in a heterogeneous population.

Discrete choice means that each consumer chooses only one out of a set of differentiated goods and is an interesting case which applies to a wide range of consumer goods, for which consumers do not have a preference for variety (for an exploration of discrete choice models of product differentiation see Anderson, de Palma, and Thisse, 1992). A consumer in my model buys only one type of good in a market depending on the relative prices between the goods. The quantity which consumers buy depends on the relevant price. Consumers are heterogeneous in three respects: 
(1) they have different critical relative prices when one good becomes more valuable than another, (2) their demand functions for each good conditional on buying the particular good differ between consumers and (3) they have different income. To model demand heterogeneity I take Grandmont's (1993) parametrization of demand. Demand heterogeneity is split into two parts: consumers have different rescaling parameters of the units of measurement compared to a base type and there exist different base types in the population. Grandmont has shown that heterogeneity of demand behavior with respect to a parameter gives rise to aggregate demand which is "close" to unit elastic demand, i.e. the price sensitivity of market expenditure is small. Note that a market in which total expenditure reacts rather insensitive to price changes makes the partial equilibrium modeling attractive. This regularity of the aggregate will turn out to be important in my model of price competition.

Since consummers can switch between the differentiated goods, total expenditure on a single differentiated goods can react very sensitive to price changes. The heterogeneity in the discrete choice between the differentiated goods is characterized by a log-concave density over switching points (following Caplin and Nalebuff, 1991b, and Dierker, 1991). This restriction is useful and encompasses a wide range of density function (see section 2). In the model strengthening the assumption on the concavity of switching parameters allows for less heterogeneous demand behavior, which means that aggregate demand is further away from the unit-elastic case. Income heterogeneity in my model will not place restrictions on aggregate demand but in contrast to models with unit demand does not lead to problems of equilibrium existence (see Peitz, 1999).

I interpret the model as a model of short-run competition in prices. The property that the differentiated goods are not perfect substitutes in the aggregate is due to the heterogeneity of switching points of the consumers. This heterogeneity can be explained by intrinsic differences in tastes (as in the literature on product differentiation, see e.g. Eaton and Lipsey, 1989), random decision making (or random utility, see e.g. Anderson, de Palma and Thisse, 1992), which may be due to heterogeneous information, or previous consumption decisions leading to heterogeneous switching costs (for other explanations of switching costs see Klemperer, 1995).

In the model, which is described in detail in section 2 , I show that there exists a pure-strategy Nash equilibrium in prices when there is heterogeneity amongst 
consumers (section 3). I present four formalizations: (1) bounded rational firms ( $\varepsilon$-maximizers), (2) bounded rational consumers (with an error in the decision rule), (3) profit maximizing firms which have to reach a minimal market share, (4) rational consumers and firms with the corresponding existence results in Theorems 1 to 4 . The main results of the paper is Theorem 4.

Under an additional distributional assumption the associated game (with bounded rational consumers) is log-supermodular and dominance solvable (section 4). Quasisupermodularity allows me to show existence which is not based on the convexvaluedness of the best response correspondence (Proposition 2). In addition, section 4 presents results on comparative statics which are implied by the properties of the profit functions: higher marginal costs and increased sales taxes imply higher equilibrium prices of all firms. Section 5 concludes.

\section{The model}

I consider a market with a finite number of differentiated goods. The set of goods is denoted by $N=\{1, \ldots, n\}$. Each good $i \in N$ has a price $p_{i}>0$. There are other goods in the economy but their prices are fixed. These other goods are captured by the composite commodity 0 which has the normalized price index $p_{0} \equiv 1$. The existence of other markets is important because market expenditure is allowed to be price-dependent. For fixed total income and no other markets this could not be the case.

\section{Consumers}

A consumer with fixed income $w>0$ has a utility function with arguments $x_{0}, x$ where $x$ is the collection of differentiated goods $x=\left(x_{i}\right)_{i \in N} \in \Re_{+}^{n}$. I consider utility functions according to which discrete choice will result. In particular, I impose that preferences in the subspace of differentiated goods are linear for any given quantity $x_{0}$ and the slope is independent of $x_{0}$.

$$
U\left(x, x_{0}\right)=u\left(\sum_{i \in N} e^{\theta_{i 1}} x_{i}, x_{0}\right)
$$

where $\theta_{i 1}$ are parameters with $\theta_{11}=0$ (see below). 
Each consumer maximizes her utility subject to her budget constraint. This can be interpreted as going through the following program. She first decides which good to buy taking only the relative prices $p_{j} / p_{i}, i, j \in N$ into account. She buys for instance good 1 and none of the other differentiated goods if $\log p_{i}-\log p_{1} \geq \theta_{1 i}$ for all $i>1$. The parameter $\theta_{i j}$ is referred to as a switching point and is the logarithmic critical price ratio at which a consumer is indifferent between goods $i$ and $j$. At prices $p$ a consumer is going to buy good $i^{\prime}$ if $i^{\prime}=\operatorname{index}\left(\max \left\{-\log p_{1},\left(\theta_{1 i}-\log p_{i}\right)_{i>1}\right\}\right)$. The set of switching parameters $\theta_{i j}, i, j, k \in N$ satisfies $\theta_{i j}=-\theta_{j i}, \theta_{k j}+\theta_{j i}=\theta_{k i}$, and $\theta_{i i}=0$. Hence, a vector $\theta_{1}=\left(\theta_{12}, \ldots, \theta_{1 n}\right)$ determines all $\theta_{i j}$, which are introduced in order to make the presentation symmetric for the goods $i \neq 1$.

At the second step, a consumer chooses according to her utility function $u$. She decides on the level of expenditure considering only the price of the good she is actually buying from.

A consumer is characterized by her utility function $u$ and slopes of the indifference curves between the differentiated goods fully determined by $\theta_{1}$. Since I want to work with demand functions which are continuous, I assume that $u$ is continuously differentiable. In addition, I assume that consumers always choose in the interior of the consumption set $\Re_{+}^{2}$ when maximizing $u$. Note that quasi-linear preferences are ruled out by this assumption. Formally, $\lim _{\widetilde{x}_{i} \rightarrow 0}\left(\partial u\left(\widetilde{x}_{i}, x_{0}\right) / \partial x_{i}\right)=\infty$ and $\lim _{\widetilde{x}_{0} \rightarrow 0}\left(\partial u\left(x_{i}, \widetilde{x}_{0}\right) / \partial x_{0}\right)=\infty$. This implies that the nonnegativity constraints $x_{0}, x_{i} \geq 0$ are automatically satisfied when maximizing the utility function $u$ subject to $p_{i} x_{i}+x_{0} \leq w$.

A consumer's budget constraint reads $p \cdot x+x_{0} \leq w$ where $p=\left(p_{i}\right)_{i \in N}$. Consumers maximize their utility

$$
\begin{aligned}
& \max _{x_{0}, x} U\left(x, x_{0}\right)=\max _{i \in N} \max _{x_{0}, x_{i}} u\left(e^{\theta_{i 1}} x_{i}, x_{0}\right) . \\
& \text { s.t. } p \cdot x+x_{0} \leq w \\
& \text { s.t. } p_{i} x_{i}+x_{0} \leq w
\end{aligned}
$$

Individual demand functions $\xi_{i}(p, w)$ for each good $i \in N$ are obtained as $\xi_{i}(p, w)= \begin{cases}\arg \max _{x_{i}} u\left(e^{\theta_{i 1}} x_{i}, w-p_{i} x_{i}\right) & \text { if } \log p_{j}-\log p_{i} \geq \theta_{i j} \text { for all } j>i \\ & \text { and } \log p_{j}-\log p_{i}>\theta_{i j} \text { for all } j<i \\ 0 & \text { else. }\end{cases}$ 
To avoid correspondences I assumed that the consumer rather buys from the good with a smaller index at a relative price equal to the switching point $\theta_{i j}$. As consumers will be assumed to be different and mass points for a distribution over the switching points will be excluded, demand can be arbitrary at the switching point without changing the result.

Consumer choice has been derived from utility maximization. As explained below, the approach is compatible with demand functions which are not derived from utility maximization.

\section{From Individual to Aggregate Demand}

Up to now every consumer was described by a collection of switching points $\theta_{1}$, an income $w>0$, and a utility function $u$. Along the lines of Grandmont (1987, 1992, 1993) I define classes or types of consumers by a specific parametrization of preferences or demand and consider heterogeneity with respect to a parameter inside such a class. ${ }^{1}$ Only differences within each class will play a role. Demand functions are parametrized as follows. A consumer is said to be of type $a \in \mathcal{A}$ if there is a rescaling parameter $\beta \in \Re$ such that $u\left(e^{-\beta} x_{1}, x_{0}\right)$ coincides with the utility function of the base consumer and if the consumer has the same income as the base consumer. In an abuse of notation I now introduce more arguments into the utility function. The utility function of a base consumer of type $a$ is written as $u\left(a, 0, x_{1}, x_{0}\right)$. The value of a utility function of a consumer with parameter $\beta$ is written as $u\left(a, \beta, x_{1}, x_{0}\right)=u\left(a, 0, e^{-\beta} x_{1}, x_{0}\right)$. Conditional demand functions are $\zeta\left(a, \beta, p_{1}\right) \equiv \arg \max _{x_{1}} u\left(a, \beta, x_{1}, w-p_{1} x_{1}\right)$. It follows that

$$
\zeta\left(a, \beta, p_{1}\right)=e^{\beta} \zeta\left(a, 0, e^{\beta} p_{1}\right)
$$

Consumers of the same type $a$ have the same conditional demand function $\zeta$ up to a rescaling of the units of measurement. Each consumer of type $a$ can now be described by a rescaling parameter $\beta$, a generating demand function $\zeta$, and parameters of switching $\theta_{1}$. Since I am only working with conditional demand functions $\zeta$ it does not really matter whether they are derived from utility maximization or reflect, for

\footnotetext{
${ }^{1}$ Such a parametrization has been introduced by Mas-Colell and Trockel (1977) and further used by Dierker, Dierker, and Trockel (1984). See in particular Grandmont (1992) for references on related literature.
} 
instance, rules of thumb. Individual demand is

$$
\xi_{i}\left(a, \beta, \theta_{i}, p\right)=\chi_{i}\left(p, \theta_{i}\right) \zeta\left(a, \beta-\theta_{i 1}, p_{i}\right)
$$

where $\theta_{i}=\left(\theta_{i 1}, \ldots, \theta_{i, i-1}, \theta_{i, i+1}, \ldots \theta_{i, n}\right)$ and $\chi_{i}$ is an indicator function defined as

$$
\chi_{i}\left(p, \theta_{i}\right)= \begin{cases}1 \quad & \text { if } \log p_{j}-\log p_{i} \geq \theta_{i j} \text { for all } j>i \\ & \text { and } \log p_{j}-\log p_{i}>\theta_{i j} \text { for all } j<i \\ 0 & \text { else }\end{cases}
$$

Consumers of the same income are heterogeneous in two respects. They have different switching points and their demand functions are different. Assumptions on the population are formally stated for later reference. (A.1) contains assumptions on distributions over the switching points $\left(\theta_{i j}\right)_{j \in N, j \neq i}$. Denote $\Theta_{i}=\times_{j \in N, j \neq i}\left[\underline{\theta}_{i j}, \bar{\theta}_{i j}\right]$.

- (A.1). For all $i \in N$ : there exist continuous distribution functions $G_{i}$ over $\theta_{i} \in \Re^{n-1}$ with $G_{i}(0)=1 / n$. $G_{i}$ has a density $g_{i}$ which is positive and continuously differentiable on int $\Theta_{i} . g_{i}$ has bounded support $\Theta_{i}$, i.e. $\theta_{i j}<\underline{\theta}_{i j}$ for some $j \in N, j \neq i$ implies $G_{i}\left(\theta_{i}\right)=0$, and $\theta_{i j}>\bar{\theta}_{i j}$ for all $j \in N, j \neq i$ implies $G_{i}\left(\theta_{i}\right)=1$. $g_{i}$ and $\partial g_{i}\left(\theta_{i}\right) / \partial \theta_{i j}, j \neq i$, are uniformly bounded from above on int $\Theta_{i}$.

For convenience, I assume that $G_{i}(0)=1 / n$ which is not restrictive because I am free to choose the units of measurement of the differentiated goods. I want to work with twice continuously differentiable profit functions. For this reason the differentiability assumptions are made. The assumption that $g_{i}$ is positive on int $\Theta_{i}$ implies that $\left(\partial / \partial \theta_{i j}\right) G_{i}\left(\theta_{i}\right) \neq 0$ for all $j \neq i$. Hence, I will analyze a model of "global competition", i.e. a price change of a differentiated good has an effect on the demand of all other differentiated goods.

I assume that $g_{i}, i \in N$ has a bounded support because I need that mean expenditure on a good turns sufficiently fast to zero for its corresponding price turning to infinity while holding the other prices fixed. The assumption of a bounded support says that for given prices $p_{-i}$ one can always find a price $p_{i}$ sufficiently large such that mean expenditure on good $i$ is equal to zero. I make use of this assumption in order 
to establish bounds for the price-sensitivity of mean expenditure and in order to construct compact strategy sets. For the latter it is convenient but not necessary (see Caplin and Nalebuff, 1991b, for a result with unbounded support). If the intervals $\left[\underline{\theta}_{i j}, \bar{\theta}_{i j}\right]$ degenerated to a single point all consumers would be identical with respect to the switching point. In such a case also mean expenditure is discontinuous; it is the Bertrand case with homogeneous goods. On the other hand, if, for all $i \in N, g_{i}$ had unbounded support there would be a positive demand left for any price combination $p$ and each good with its index in $N$. The assumption of a rectangular support is made for convenience; it is only important that the support is convex. Note that for any price vector $p, \sum_{i \in N} G_{i}=1$. Denote $\theta_{i}=\left(\theta_{i j}, \theta_{i,-j}\right) . \partial g_{i}\left(\underline{\theta}_{i j}, \theta_{i,-j}\right) / \partial \theta_{i j}$ is defined as $\lim _{\theta_{i j} \backslash \underline{\theta}_{i j}} \partial g_{i}\left(\theta_{i}\right) / \partial \theta_{i j}$ and $\partial g_{i}\left(\bar{\theta}_{i j}, \theta_{i,-j}\right) / \partial \theta_{i j} \equiv \lim _{\theta_{i j} / \bar{\theta}_{i j}} \partial g_{i}\left(\theta_{i}\right) / \partial \theta_{i j}$. Partial derivatives of $G_{i}$ are defined accordingly on the boundary.

Important for the calculations is stochastic independence of the three components describing the population of consumers.

- (A.2). $a, \beta$, and $\theta_{1}$ are stochastically independent.

(A.2) implies that consumers in a particular segment of the differentiated market, formalized by $\theta_{1}$, do not systematically differ from the rest of the population in their expenditure functions in the market. The next assumption is made in order to integrate over individual demand functions.

- (A.3).

(1) $\mathcal{A}$ is a separable metric space of types, $\mu$ is a probability measure on $\mathcal{A}$.

(2) For each type $a$ there exists a conditional distribution over $\beta$ with density $f$.

(3) Income $w^{a}>0$ depends continuously on type $a$ and average income is finite, i.e.

$$
W=\int_{\mathcal{A}} w^{a} \mu(d a)<\infty
$$


Under (A.1)-(A.3) mean demand of good $i$ reads

$$
\begin{aligned}
X_{i}(p) & =\int_{\Theta_{i}} \int_{\Re} \int_{\mathcal{A}} \chi_{i}\left(p, \theta_{i}\right) \zeta\left(a, \beta-\theta_{i 1}, p_{i}\right) \mu(d a) f(\beta) d \beta g_{i}\left(\theta_{i}\right) d \theta_{i} \\
& =\frac{1}{p_{i}} \int_{\times_{j \in N, j \neq i}\left[\underline{\theta}_{i j}, \log p_{j}-\log p_{i}\right]} B\left(p_{i}, \theta_{i 1}\right) g_{i}\left(\theta_{i}\right) d \theta_{i}
\end{aligned}
$$

where

$$
B\left(p_{i}, \theta_{i 1}\right) \equiv \int_{\Re} \int_{\mathcal{A}} p_{i} e^{\beta-\theta_{i 1}} \zeta\left(a, 0, e^{\beta-\theta_{i 1}} p_{i}\right) \mu(d a) f(\beta) d \beta
$$

denotes conditional mean expenditure of type $\theta_{1}$. It will be important to show that conditional mean expenditure reacts slowly to a price change. In the work of Dierker (19991) and Caplin and Nalebuff (1991b, subsection 8.2) it was assumed that conditional mean expenditure is a constant. In this paper I do not make such an assumption or any shape assumptions on individual demand functions (for a discussion see the conclusion).

Remark 1 jFrom the definition of individual demand it follows that conditional mean expenditure $B\left(p_{i}, \theta_{i 1}\right)$ only depends on $\log p_{i}-\theta_{i 1}$, i.e.

$$
\frac{\partial B\left(p_{i}, \theta_{i 1}\right)}{\partial \theta_{i 1}}+\frac{\partial B\left(p_{i}, \theta_{i 1}\right)}{\partial \log p_{i}}=0
$$

\section{Firms}

Firm behavior is standard: good $i, i \in N$, is produced by firm $i$ with constant marginal $\operatorname{costs} c_{i}>0$. Each firm faces a mean demand function $X_{i}(p)$ depending on the prices in the market. Each firm is a price setter. The strategic variable, price $p_{i}$, is chosen as the best response to the prices of the other firms. For prices above marginal costs firms will satisfy demand. Profits are given as

$$
\pi_{i}(p)=\left(p_{i}-c_{i}\right) X_{i}(p), i \in N
$$

\section{Equilibrium}

In the paper I look at pure-strategy Nash equilibria in prices. A pure strategy Bertrand-Nash equilibrium is a vector of prices $p^{*} \in \Re_{++}^{n}$ such that for all $i \in N$

$$
\pi_{i}\left(p_{i}^{*}, p_{-i}^{*}\right) \geq \pi_{i}\left(p_{i}, p_{-i}^{*}\right) \quad \text { for all } p_{i} \in \Re_{++}
$$

In other words, $p_{i}^{*}$ is an element of the best response correspondence for $p_{-i}^{*}$. 


\section{Equilibrium existence and demand heterogene- ity}

The main problem to show existence in models of price competition is to establish the quasi-concavity of profit functions. I show this property to hold on a compact set of prices and prove that firms never set prices outside this set. In particular, I show that heterogeneous demand behavior implies that mean expenditure $B\left(p_{i}, \theta_{i 1}\right)$ does not depend strongly on its price.

Inserting mean demand into the profit functions gives profit functions which depend on prices and the characteristics of the market. It will be helpful to consider logarithmic profits. Denote $B_{0}\left(p_{i}\right)=B\left(p_{i}, 0\right)$.

$$
\begin{aligned}
\log \pi_{i}(p)= & \log \frac{p_{i}-c_{i}}{p_{i}}+\log G_{i}\left(\left(\log p_{j}-\log p_{i}\right)_{j \in N, j \neq i}\right)+\log B_{0}\left(p_{i}\right) \\
& +\log \frac{\int_{\times_{j \in N, j \neq i}\left[\underline{\theta}_{i j}, \log p_{j}-\log p_{i}\right]} B\left(p_{i}, \theta_{i 1}\right) g_{i}\left(\theta_{i}\right) d \theta_{i}}{B_{0}\left(p_{i}\right) G_{i}\left(\left(\log p_{j}-\log p_{i}\right)_{j \in N, j \neq i}\right)}
\end{aligned}
$$

The profit function consists of four additive terms. The first existence result shows that an oligopoly with profit functions consisting of the first two terms has an equilibrium and that the last two terms are negligible under sufficient heterogeneity. The second result interprets profit functions consisting of the first three additive terms as the presented model with the only difference that consumers are bounded rational. The third and fourth result show equilibrium existence for profit functions as stated in equation (1). I will show that the respective profit functions are quasiconcave on a compact set of prices. In contrast to Dierker (1991) and Caplin and Nalebuff (1991b), in specifications 2 to 4 I cannot show that profit functions are quasi-concave for all prices so that the construction of compact strategy spaces is of particular importance.

The outline of this section then is as follows: the first theorem establishes equilibrium existence with bounded rational firms (subsection 3.1), the second equilibrium with bounded rational consumers (subsection 3.2), the third existence result holds for profit maximizing firms which have to satisfy a minimal market share and the fourth and main result allows for fully rational consumers and firms without restrictions (both subsection 3.3). Theorems 1 to 4 say that if the densities over 
switching parameters are log-concave and if demand behavior is heterogeneous then there exists an equilibrium.

\subsection{Bounded rational firms}

Considering the four additive terms of the profit function (1) I start with the first term which satisfies the following concavity property:

Remark 2 The first term $\log \left(\left(p_{i}-c_{i}\right) / p_{i}\right)$ is concave in $\log p_{i}$ for all $p_{i}>c_{i}$. The second derivative with respect to $\log p_{i}$ is bounded from above by some negative number when prices are chosen from a compact strategy space.

The fact of the negative upper bound of the second derivative on a compact set will be important below. Because of Remark 2 one only has to worry about the second to fourth term in the profit function (1). In this paper two kinds of heterogeneity will be important. First, heterogeneity with respect to $\theta_{i}$ implies that consumers have different relative prices at which they are indifferent between a pair of goods. Second, heterogeneity with respect to $u$, formalized by a distribution over $\beta$, will be crucial in making expenditure less price-sensitive in the aggregate than it is on the individual level.

First, I make an assumption on the heterogeneity of $\theta_{i}$ in order to take care of the second term in (1).

- (A.4). $g_{i}$ is log-concave in $\theta_{i}, i \in N$.

In particular Caplin and Nalebuff (1991a) discuss which distributions have logconcave densities. They also give the relevant references. For example the Normal and, with parameter restrictions, the multivariate Beta distribution have log-concave densities. Results carry over to truncations with convex support.

Following the work of Dierker (1991) and Caplin and Nalebuff (1991b) also the second term is log-concave in logarithmic price if the density has this property. This 
result is stated as Lemma 1 (for the proof see the appendix.). One of the aggregation theorems of Prékopa (1973) underlies the result. Dierker (1991) and Caplin and Nalebuff (1991b) were to my knowledge the first who applied the aggregation results of Prékopa and Borell to models of imperfect competition.

Lemma 1 Assume (A.1),(A.4). $G_{i}\left(\left(\log p_{j}-\log p_{i}\right)_{j \neq i}\right)$ is log-concave in $\log p_{i}$.

In this section I analyze profit functions of the form (1) looking for a generalized version of Nash equilibrium. A pure strategy Bertrand-Nash $\varepsilon$-equilibrium is a vector of prices $p^{*} \in \Re_{++}^{n}$ such that for all $i \in N$

$$
\pi_{i}\left(p_{i}^{*}, p_{-i}^{*}\right) \geq \pi_{i}\left(p_{i}, p_{-i}^{*}\right) \frac{1}{1+\varepsilon} \quad \text { for all } p_{i} \in \Re_{++}
$$

Firms are not maximizers but do not bother to change their strategy if such a deviation increases profits by a factor $\varepsilon$ or less. Firms which are $\varepsilon$-maximizers can be labelled bounded rational. The reason for not fully maximizing profits can be motivated by measurement errors such as accounting errors (which are proportional to the level of profit). In the pure Bertrand model Baye and Morgan (1996) obtain results on $\varepsilon$-equilibria (with additive $\varepsilon$ ).

Consequently, equilibrium existence for profit functions, which are the sum of the first and the second term, implies the existence of $\varepsilon$-equilibria if the third and the fourth term in (1) can be made arbitrarily small. As will be stated by the following two lemmas this is implied by sufficient heterogeneity with respect to $\beta$.

Assumption (A.5) says that there is a strict lower bound of expenditure $B\left(p_{i}, \theta_{i 1}\right)$ for all $\theta_{i 1} \in\left[\underline{\theta}_{i 1}, \bar{\theta}_{i 1}\right]$. This means that the aggregate of consumers with a particular switching parameter spends a positive minimum budget share in the differentiated market. A similar assumption is also made in Grandmont $(1992,1993)$ and is needed in the proofs of several lemmata.

- (A.5). There exists $\delta>0$ such that $B\left(p_{i}, \theta_{i 1}\right) \geq \delta W$ for all $p_{i}>0$ and $\theta_{i 1} \in\left[\underline{\theta}_{i 1}, \bar{\theta}_{i 1}\right], i \in N, i>1$. 
If there was only one type $(a, \beta=0)$ in the market, conditional demand of the form

$$
\zeta\left(a, 0, p_{1}\right)=\frac{\delta(a) w}{p_{1}}+z\left(a, 0, p_{1}\right)
$$

$\delta(a)>0, z: \mathcal{A} \times \Re \times \Re_{++} \longrightarrow \Re_{+}$, implies under (A.4) that there is a lower bound on individual expenditure for all switching parameters from $\Theta$. Given a type space $\mathcal{A}$ (A.5) is satisfied if (A.4) holds and if there is a subspace $\mathcal{A}_{1} \subseteq \mathcal{A}$ of positive measure such that the above equation of conditional demand holds.

With (A.6) I assume uniform integrability over $\beta$. The average slope in absolute value $m_{1}$ serves as a measure of heterogeneity, $0<m_{1}<\infty$ for distributions with unbounded support. A small $m_{1}$ stands for a "flat" distribution and thus for a large heterogeneity of demand with respect to the parameter $\beta$ (see Kneip, 1993).

- (A.6). $f$ is continuously differentiable with $f^{\prime}$ uniformly integrable, i.e. there exists a real number $m_{1}$ such that

$$
\int_{\Re}\left|f^{\prime}(\beta)\right| d \beta=m_{1}<\infty .
$$

The next lemma puts an arbitrarily small bound on the price elasticity of mean expenditure $B_{0}\left(p_{i}\right)$.

Lemma 2 Under (A.1),(A.2),(A.5),(A.6) the following inequality holds

$$
\left|\frac{\partial \log B_{0}\left(p_{i}\right)}{\partial \log p_{i}}\right| \leq \frac{m_{1}}{\delta} .
$$

\section{Proof.}

$$
B_{0}\left(p_{i}\right)=\int_{\Re} \int_{\mathcal{A}} p_{i} e^{\beta} \zeta\left(a, 0, e^{\beta} p_{i}\right) \mu(d a) f(\beta) d \beta .
$$

Substitute as in Grandmont (1993) $r=\beta+\log p_{i}$

$$
\begin{aligned}
B_{0}\left(p_{i}\right) & =\int_{\Re} \int_{\mathcal{A}} e^{r} \zeta\left(a, 0, e^{r}\right) \mu(d a) f\left(r-\log p_{i}\right) d r \\
\frac{d B_{0}\left(p_{i}\right)}{d \log p_{i}} & =-\int_{\Re} \int_{\mathcal{A}} e^{r} \zeta\left(a, 0, e^{r}\right) \mu(d a) f^{\prime}\left(r-\log p_{i}\right) d r
\end{aligned}
$$




$$
\begin{aligned}
& =-\int_{\Re} \int_{\mathcal{A}} p_{i} \zeta\left(a, \beta, p_{i}\right) \mu(d a) f^{\prime}(\beta) d r \\
\left|\frac{d B_{0}\left(p_{i}\right)}{d \log p_{i}}\right| & \leq m_{1} W \\
\left|\frac{d \log B_{0}\left(p_{i}\right)}{d \log p_{i}}\right| & \leq m_{1} \frac{W}{B_{0}\left(p_{i}\right)} \leq \frac{m_{1}}{\delta} .
\end{aligned}
$$

The lemma implies that $\left|\log B_{0}\left(p_{i}^{\prime}\right)-\log B_{0}\left(p_{i}\right)\right|$ can be made arbitrarily small on a compact set of prices if the average absolute value of the slope of the density is small, i.e. if $m_{1}$ is small. When price $p_{i}$ is chosen from a compact strategy set $\left[c_{i}, z\right]$ then, for firm $i$, deviations from equilibrium profits are bounded above by the factor $\left(z-c_{i}\right)\left(m_{1} / \delta\right)$ when only the third term is taken into account.

The last term of profit function (1) is rewritten as

$$
\log \left(1+\frac{\int_{\times_{j \in N, j \neq i}\left[\underline{\theta}_{i j}, \log p_{j}-\log p_{i}\right]}\left(B\left(p_{i}, \theta_{i 1}\right)-B_{0}\left(p_{i}\right)\right) g_{i}\left(\theta_{i}\right) d \theta_{i}}{B_{0}\left(p_{i}\right) G_{i}\left(\left(\log p_{j}-\log p_{i}\right)_{j \in N, j \neq i}\right)}\right) \equiv \log (1+R)
$$

which implicitly defines $R$. This term can be made arbitrarily small if $R$ is close to zero.

Lemma 3 Under (A.1),(A.2),(A.5),(A.6). Then $R$ can be bounded in absolute value by any positive number if $m_{1}$ is sufficiently small, in particular, the bound $\left(\bar{\theta}_{i 1}-\underline{\theta}_{i 1}\right) m_{1} / \delta$ is never broken by firm $i$.

Proof. Since by (A.5) $B_{0}\left(p_{i}\right)$ is bounded from below it is sufficient to show that $\left|B\left(p_{i}, \theta_{i 1}\right)-B_{0}\left(p_{i}\right)\right|$ can be made arbitrarily small. Because of Remark 1, (A.1), (A.2), (A.5), and (A.6) imply that

$$
\left|\frac{\partial \log B\left(p_{i}, \theta_{i 1}\right)}{\partial \theta_{i 1}}\right| \leq m_{1} W .
$$

Consequently,

$$
\begin{aligned}
\left|B\left(p_{i}, \theta_{i 1}\right)-B_{0}\left(p_{i}\right)\right| & \leq\left|\int_{0}^{\theta_{i 1}}\right| \frac{\partial \log B\left(p_{i}, t\right)}{\partial t}|d t| \\
& \leq\left|\theta_{i 1}\right| m_{1} W
\end{aligned}
$$


Since $\theta_{i 1}$ has compact support this term can be made arbitrarily small. An explicit bound depending on the measure of heterogeneity is derived by the following

$$
\begin{aligned}
|R| & \leq \frac{\int_{\times_{j \in N, j \neq i}\left[\underline{\theta}_{i j}, \log p_{j}-\log p_{i}\right]}\left|\theta_{i 1}\right| m_{1} W g_{i}\left(\theta_{i}\right) d \theta_{i}}{\delta W G_{i}\left(\left(\log p_{j}-\log p_{i}\right)_{j \in N, j \neq i}\right)} \\
& \leq \frac{m_{1}}{\delta} \frac{\int_{\times_{j \in N, j \neq i}\left[\underline{\theta}_{i j}, \log p_{j}-\log p_{i}\right]}\left(\bar{\theta}_{i 1}-\underline{\theta}_{i 1}\right) g_{i}\left(\theta_{i}\right) d \theta_{i}}{G_{i}\left(\left(\log p_{j}-\log p_{i}\right)_{j \in N, j \neq i}\right)} \\
& =\left(\bar{\theta}_{i 1}-\underline{\theta}_{i 1}\right) \frac{m_{1}}{\delta} .
\end{aligned}
$$

On a compact set of prices Lemma 1 is used to show the existence of an equilibrium for profit functions consisting of terms 1 and 2. With Lemmas 2 and 3 one can show that such an equilibrium is an $\varepsilon$-equilibrium. In the proof I have to show that firms will choose from a compact set of prices. In order to show this I use that the switching parameters have a compact support. I define

$$
\log z \equiv \max \left\{\left(\bar{\theta}_{i j}-\underline{\theta}_{i j}+\log \frac{n}{n-1}+\log c_{i}\right)_{i, j \in N, j \neq i}\right\}
$$

Theorem 1 says that under the above assumptions an $\varepsilon$-equilibrium exists if consumers are sufficiently heterogeneous with respect to their conditional demand functions.

Theorem 1 Assume (A.1)-(A.4). There exists an equilibrium for profit functions $\pi_{i}(p)=\left(\left(p_{i}-c_{i}\right) / p_{i}\right) G_{i}\left(\left(\log p_{j}-\log p_{i}\right)_{j \neq i}\right)$. Assume in addition (A.5) and (A.6). For any $\varepsilon>0$ there exists a pure-strategy Bertrand-Nash $\varepsilon$-equilibrium if $m_{1}$ is sufficiently small.

Proof. First, equilibrium existence for profit functions as the sums of the first and second additive term of the right-hand side of (1) is shown for given compact strategy sets. Lemmata 1 and 4 (the latter in the Appendix) then say that these profit functions are quasi-concave for given compact strategy sets. In Lemma 5 Kakutani's fixed point theorem is applied for given compact strategy sets. Lemma 6 shows that firms will always choose out of these strategy sets. Lemmata 4 to 6 are delegated to the appendix. For compact strategy sets Lemma 2 implies that the 
third term of differences of logarithmic profit function (1) can be made arbitrarily small for $m_{1}$ sufficiently small. Lemma 3 implies that differences in logarithmic profit functions of the fourth term are arbitrarily small for $m_{1}$ sufficiently small. Hence, by Lemmata 2 and 3 an equilibrium strategy for profit functions as the sums of the first and second term of (1) are $\varepsilon$-maximal, $\varepsilon>0$, for $m_{1}$ sufficiently small: Denote equilibrium profits of firm $i$ with $\pi_{i}^{*}$ and profits after a price change $\pi_{i}^{\prime}$, denote $\log z^{\prime}=\log z-\max _{j \neq i} \underline{\theta}_{i j}$. Since $p_{i}^{*} \leq z$ a deviation to $p_{i}^{\prime} \geq z^{\prime}$ cannot be profitable. For $p_{i}^{\prime}<z^{\prime}, \log \pi_{i}^{\prime}-\log \pi_{i}^{*} \leq\left(z^{\prime}-c_{i}\right)\left(m_{1} / \delta\right)-2 \log \left(1-\left(\bar{\theta}_{i 1}-\underline{\theta}_{i 1}\right)\left(m_{1} / \delta\right)\right) \leq \log (1+\varepsilon)$ for $m_{1}$ sufficiently small because $\log (1+|R|)<|R|<-\log (1-|R|)$.

\subsection{Bounded rational consumers}

At this point I present and discuss an alternative formulation of consumer behavior which is not fully rational but may be called bounded rational. Consumers buy according to the same conditional demand function $\zeta\left(p_{i}, w\right)$ independent of the switching parameters $\theta$.

Each consumer splits the decision problem into two parts (two stage decision problem): first how much to demand of a good in the differentiated market and second to decide which of the differentiated goods to buy. ${ }^{2}$ Ex ante goods are identical, i.e. consumers maximize $u\left(x_{0}, \sum_{i=1}^{n} x_{i}\right)$ under their budget constraint. Their demand without an error is $\xi_{i}(p, w)=\zeta\left(p_{i}, w\right)$ if $p_{i}<p_{j}$, for all $j \neq i$, and $\xi_{i}(p, w)=0$ if $p_{i}>p_{j}$, for some $j \neq i$. Introducing a vector of errors $e_{i}, i \in N$, which is drawn independently across consumers from a probability distribution with compact support, generates preferences for a particular differentiated good. If the realization of $e_{i}>0$, a consumer is willing to pay a higher price for the good than without error. Consumers do not make systematic errors if $E e_{i}=0$ for all $i \in N$. Define switching points $\theta_{i 1}=e_{1}-e_{i}$. Demand with the error included is assumed to be of the form $\xi_{i}(p, w)=\zeta\left(p_{i}, w\right)$ if $\log p_{i}<\log p_{j}-\theta_{i j}$, for all $j \neq i$, and $\xi_{i}(p, w)=0$ if $\log p_{i}>\log p_{j}-\theta_{i j}$, for some $j \neq i$. This means that consumers derive demand for good $i$ conditional on buying none of the other differentiated goods from

\footnotetext{
${ }^{2}$ Note that this corresponds to the two-stage budgeting in applied demand analysis (see e.g. Deaton and Muellbauer, 1980).
} 
maximizing $u\left(x_{i}, x_{0}\right)$ s.t. $p_{i} x_{i}+x_{0} \leq w$ but make an error in the decision which good to buy, i.e. they do not necessarily buy the cheapest one in the market. This may be interpreted as a consumer being imperfectly informed about prices when making the decision which good to buy but once this decision is made (trip to the firm's factory) the consumer observes perfectly the price of the good chosen and maximizes utility. Errors in the decision rule have been discussed in the literature e.g. in Tversky (1972). My formulation leads to profit functions

$$
\log \pi_{i}=\log \frac{p_{i}-c_{i}}{p_{i}}+\log B_{0}\left(p_{i}\right)+\log G_{i}\left(\left(\log p_{j}-\log p_{i}\right)_{j \neq i}\right) .
$$

- (A.7). $f$ is twice continuously differentiable with $f^{\prime}$ and $f^{\prime \prime}$ uniformly integrable, i.e. there exists a minimal real number $m_{2}$ such that

$$
\begin{aligned}
\int_{\Re}\left|f^{\prime}(\beta)\right| d \beta & \leq m_{2}<\infty \\
\text { and } \int_{\Re}\left|f^{\prime \prime}(\beta)\right| d \beta & \leq m_{2}<\infty .
\end{aligned}
$$

Under this assumption which is stronger than (A.6), $m_{2}$ serves as the measure of demand heterogeneity. The following lemma provides bounds for the price sensitivity of mean expenditure (expressed as elasticity and sensitivity of the elasticity).

Lemma 7 Assume (A.1)-(A.3),(A.5),(A.7). The following inequalities hold

$$
\begin{aligned}
\left|\frac{d \log B_{0}\left(p_{i}\right)}{d \log p_{i}}\right| & \leq \frac{m_{2}}{\delta} \\
\left|\frac{d^{2} \log B_{0}\left(p_{i}\right)}{\left(d \log p_{i}\right)^{2}}\right| & \leq \frac{m_{2}}{\delta}\left(1+\frac{m_{2}}{\delta}\right) .
\end{aligned}
$$

Proof. As in the proof of Lemma 2 I substitute $r=\beta+\log p_{i}$ in the expression of $B_{0}\left(p_{i}\right)$. Taking first and second derivatives as in Grandmont (1993) gives

$$
\begin{aligned}
\left|\frac{d B_{0}\left(p_{i}\right)}{d \log p_{i}}\right| & \leq m_{2} W \\
\left|\frac{d^{2} B_{0}\left(p_{i}\right)}{\left(d \log p_{i}\right)^{2}}\right| & =\int_{\Re} \int_{\mathcal{A}} e^{r} \zeta\left(a, 0, e^{r}\right) \mu(d a) f^{\prime \prime}\left(r-\log p_{i}\right) d r \leq m_{2} W .
\end{aligned}
$$


Consequently,

$$
\begin{aligned}
\left|\frac{d \log B_{0}\left(p_{i}\right)}{d \log p_{i}}\right| & \leq \frac{m_{2}}{\delta} \\
\left|\frac{d^{2} \log B_{0}\left(p_{i}\right)}{\left(d \log p_{i}\right)^{2}}\right| & =\left|\frac{1}{B_{0}\left(p_{i}\right)} \frac{d^{2} B_{0}\left(p_{i}\right)}{\left(d \log p_{i}\right)^{2}}-\frac{1}{B_{0}\left(p_{i}\right)^{2}}\left(\frac{d B_{0}\left(p_{i}\right)}{d \log p_{i}}\right)^{2}\right| \\
& \leq \frac{m_{2}}{\delta}\left(1+\frac{m_{2}}{\delta}\right) \cdot \square
\end{aligned}
$$

Theorem 2 Assume (A.1)-(A.5),(A.7). In the model with profit functions (2) there exists a pure-strategy Bertrand-Nash equilibrium if $m_{2}$ is sufficiently small.

Sketch of the proof. Again I have to show that firms will choose from a compact set of prices. In order to show this in Lemma 8 in the appendix, I use that mean expenditure is bounded, that $m_{2}$ is sufficiently small, and that the switching parameters have a compact support. I need that $m_{2}$ is sufficiently small in the case that there does not exist a price $p_{i}$ above which the mean expenditure function $B_{0}$ is monotone. In the case of monotonicity above some price level, I can do without $m_{2}$ being small and bound differences in mean expenditure due to the convergence of mean expenditure (which follows from the bounds of mean expenditure). By Remark 2 the second derivative in logarithmic price of the first term of (2) is bounded by some negative number. Hence, since by Lemma 7 the second derivative of the third term can be made arbitrarily small in absolute value one can show the quasi-concavity of profit functions for $m_{2}$ sufficiently small and $g$ log-concave on the compact set of prices from which firms will choose according to Lemma 8 in the appendix.

Note that in the construction of compact strategy sets (Lemma 8) I use the condition that $m_{2}$ is small. This allows me to consider the same compact strategy set as in the previous subsection. From the argument in part (ii) of the proof of Proposition 2 below it follows that it is not necessary to assume that $m_{2}$ is small in order to construct compact strategy sets.

Remark 3 At this point it seems to be appropriate to discuss the trade-off between the two different kinds of heterogeneity. As stated in Remark 2 there exists a negative 
number such that $\left.\partial^{2} \log \left(p_{i}-c_{i}\right) / p_{i}\right) /\left(\partial \log p_{i}\right)^{2}$ is bounded from above by that number on a compact set. Note that this makes it possible to show equilibrium existence even if $G_{i}$ is not log-concave. With profit functions (2) one can show equilibrium existence as long as there exists some $\kappa>0$ such that

$$
\frac{\partial^{2} \log \frac{p_{i}-c_{i}}{p_{i}}}{\left(\partial \log p_{i}\right)^{2}}+\frac{\partial^{2} \log G_{i}}{\left(\partial \log p_{i}\right)^{2}} \leq-\kappa
$$

on the compact set $\times_{i \in N}\left[c_{i}, z\right]$ and $m_{2}$ sufficiently small, i.e.

$$
\frac{m_{2}}{\delta}\left(1+\frac{m_{2}}{\delta}\right)<\kappa
$$

Denote the set of prices $P=\times_{i \in N}\left[c_{i}, z\right] \cap\left\{\left(p_{i}\right)_{i \in N} \mid \log p_{j}-\log p_{i} \leq \bar{\theta}_{i j}\right\}$ and denote the Hessian of $\log G_{i}$ with $H_{i} \equiv\left(\partial_{i j} G_{i}\right)$ and $\iota \equiv(1, \ldots, 1)^{T} \in \Re^{n-1}$. Note that $H_{i}$ is negative semi-definite if and only if $\log G_{i}$ concave. The negative semidefiniteness of $H_{i}$ implies that $\sum_{i} \sum_{j} \partial_{i j} \log G_{i}=\iota^{T} H_{i} \iota \leq 0 . G_{i}$ is called $\tilde{\kappa}-$ strict log-concave if for the Hessian $H_{i}$ of $\log G_{i}$ the inequality $\iota^{T} H_{i} \iota \leq-\tilde{\kappa}$ holds.

Remark 4 If the second derivative of $\log G_{i}$ in $\log p_{i}$ is sufficiently negative then conditional mean expenditure is allowed to react more sensitive on prices, i.e. $m_{2}$ is not necessarily "small". Hence in order to allow for less demand heterogeneity ( $m_{2}$ not "small") $G_{i}$ has to be $\tilde{\kappa}$-strict log-concave. For $\tilde{\kappa}$ big, the inequality cannot be satisfied for densities $g_{i}$ over $\theta_{i}$ if their supports are "large". Restricting the support implies that consumers are more homogeneous with respect to their switching parameters. In addition to the possibility of a big $\tilde{\kappa}$, a smaller support of $\theta_{i}$ makes the price setting more competitive and the compact set of prices $P$ smaller. Hence, also the second derivative of the price-cost margin (in logarithms) can be bounded from above by a negative number which is greater in absolute value as the support is reduced.

Remark 5 In a duopoly there exist $\tilde{\kappa}>0$ such that the assumption of log-concavity of $g_{1},(A .4)$, implies that $G_{i}$ is $\tilde{\kappa}$-strict log-concave if $g_{1}(\theta)$ is bounded from below on $[\underline{\theta}, \bar{\theta}]$, which is for example satisfied if $g_{1}$ is uniform or truncated Normal. This is shown in the appendix. 
To conclude this subsection, I summarize the previous Remarks 3 and 4 by a suggestive statement: in order to show existence of equilibrium a less diverse expenditure pattern $\left(m_{2}\right.$ bigger) has to go hand in hand with less diversity of errors in the decision rule (support of $g_{i}$ smaller). An additional result on equilibrium existence is provided in subsection 4.1 below.

\subsection{Rational firms and consumers}

In this subsection I first consider profit functions of the form (1) in which firms are profit maximizers under the constraint that they sell to a minimal share of consumers, i.e. $G_{i} \geq \epsilon$. Afterwards this restriction on the strategies is no longer imposed.

It will be useful to rewrite profit function (1).

$$
\begin{aligned}
\log \pi_{i}= & \log \frac{p_{i}-c_{i}}{p_{i}}+\log \int_{\times_{j \in N, j \neq i}\left[\underline{\theta}_{i j} \log p_{j}-\log p_{i}\right]} B\left(p_{i}, \theta_{i 1}\right) g_{i}\left(\theta_{i}\right) d \theta_{i} \\
= & \log \frac{p_{i}-c_{i}}{p_{i}}+\log B_{0}\left(p_{i}\right)+\log \left(G_{i}\left(\left(\log p_{j}-\log p_{i}\right)_{j \neq i}\right)+\right. \\
& \left.\int_{\times_{j \in N, j \neq i}\left[\underline{\theta}_{i j}, \log p_{j}-\log p_{i}\right]} \frac{B\left(p_{i}, \theta_{i 1}\right)-B_{0}\left(p_{i}\right)}{B_{0}\left(p_{i}\right)} g_{i}\left(\theta_{i}\right) d \theta_{i}\right)
\end{aligned}
$$

As is known from Lemma 7 the second derivative of the second term can be made arbitrarily small. Although $G_{i}$ is log-concave (by Lemma 1 ) the third additive term in (3) is not necessarily concave. To save some space let me denote

$$
\begin{aligned}
D\left(\log p_{i}\right) & \equiv G_{i}\left(\left(\log p_{j}-\log p_{i}\right)_{j \neq i}\right) \\
E\left(\log p_{i}\right) & \equiv \int_{\times_{j \in N, j \neq i}\left[\underline{\theta}_{i j}, \log p_{j}-\log p_{i}\right]} \frac{B\left(p_{i}, \theta_{i 1}\right)-B_{0}\left(p_{i}\right)}{B_{0}\left(p_{i}\right)} g_{i}\left(\theta_{i}\right) d \theta_{i} .
\end{aligned}
$$

In order to show that profit functions are log-concave on a compact support I have to show that second derivative of $\log \left(D\left(q_{i}\right)+E\left(q_{i}\right)\right), q_{i} \equiv \log p_{i}$, can be bounded from above by some positive number arbitrarily close to 0 . Hence it has to be shown 
that for any $\epsilon>0$ there exists an $m_{2}$ such that

$$
\frac{1}{\left(D\left(q_{i}\right)+E\left(q_{i}\right)\right)^{2}}\left(\left(\frac{d^{2} D\left(q_{i}\right)}{d q_{i}^{2}}+\frac{d^{2} E\left(q_{i}\right)}{d q_{i}^{2}}\right)\left(D\left(q_{i}\right)+E\left(q_{i}\right)\right)-\left(\frac{d D\left(q_{i}\right)}{d q_{i}}+\frac{d E\left(q_{i}\right)}{d q_{i}}\right)^{2}\right)<\epsilon .
$$

(A.4) implies that $\left(d^{2} D\left(q_{i}\right) / d q_{i}^{2}\right) D\left(q_{i}\right)-\left(d D\left(q_{i}\right) / d q_{i}\right)^{2} \leq 0$. Consequently, under (A.4) it has to be shown that

$$
\begin{aligned}
& \frac{d^{2} E\left(q_{i}\right)}{d q_{i}^{2}}\left(D\left(q_{i}\right)+E\left(q_{i}\right)\right)+\frac{d^{2} D\left(q_{i}\right)}{d q_{i}^{2}} E\left(q_{i}\right)-2 \frac{d D\left(q_{i}\right)}{d q_{i}} \frac{d E\left(q_{i}\right)}{d q_{i}}-\left(\frac{d E\left(q_{i}\right)}{d q_{i}}\right)^{2} \\
< & \epsilon\left(D\left(q_{i}\right)+E\left(q_{i}\right)\right)^{2} .
\end{aligned}
$$

Without a positive lower bound on $D\left(q_{i}\right)$ the right-hand side of this inequality cannot be bounded from below by some positive number. This implies that without further assumptions one cannot find, for all $\epsilon>0$, an $m_{2}$ (depending on $\epsilon$ ) such that this inequality is satisfied for all prices in $P$. As shown in Lemma 9 in the appendix, if there is a positive lower bound for $D\left(q_{i}\right)$ one can make the left-hand side arbitrarily small and bound the right-hand from below by some positive number.

With a minimal market share I obtain a positive lower bound for $D\left(q_{i}\right)$. Imposing a minimal market share is appropriate in markets in which firms have to have a critical mass in order to survive. Results are unaffected when instead of consumer mass, expenditure shares, i.e. $p_{i} X_{i} /\left(\sum_{j} p_{j} X_{j}\right) \geq \epsilon$, or demand shares are considered. One justification can be that firms are run by managers and managerial incentives include minimal market share (due to dynamic considerations). Such markets also include markets with strong network externalities where there exists a critical market size below which consumers do not find the product useful. ${ }^{3}$

If the cost differences of the firms are sufficiently similar with respect to the consumer tastes, there exist minimal market share such that firms can make profit whatever the prices (above marginal costs) of the competitors are.

\footnotetext{
${ }^{3}$ This motivation leads to problems when formalized: the profit function of low price firms would need modifications because these firms can push a competitor below critical size and thus discontinuously increase their demand. Hence in order to translate my result into a model with network externalities the discontinuity must be sufficiently small and sufficiently distant from any equilibrium price vector.
} 
- (A.8) There exists a $\varphi>0$ such that

$$
\log c_{j}-\log c_{i} \in\left(\underline{\theta}_{i j}+\varphi, \bar{\theta}_{i j}-\varphi\right), i, j \in N, i<j
$$

Theorem 3 Assume (A.1)-(A.5),(A.7),(A.8). There exist minimal market shares such that the model with minimal market shares has a pure-strategy Bertrand-Nash equilibrium in which all firms are active if $m_{2}$ is sufficiently small.

Sketch of Proof. Similar to the one of Theorem 2. (A.8) implies that all firms are active in any candidate equilibrium and can reach some minimal budget shares $\epsilon_{i}$ given prices above marginal costs by the competitors, $G_{i}\left(\log c_{j}-\log c_{i}\right)=\epsilon_{i} \geq$ $\left.G_{i}\left(\underline{\theta}_{i j}+\varphi\right)_{j \neq i}\right)$. Due to Remark 2 and Lemmata 4, 7, and 9 (in the Appendix) one can show the quasi-concavity of profit function on a compact set of prices if $m_{2}$ is sufficiently small. In order to bound the second derivative of $\log \left(D\left(q_{i}\right)+E\left(q_{i}\right)\right)$ by a positive number arbitrarily small on a compact set for $m_{2}$ sufficiently small I need the log-concavity of $G_{i}$, arbitrarily small bounds on differences $\left|B\left(p_{i}, \theta_{i 1}\right)-B_{0}\left(p_{i}\right)\right|$, and first and second derivatives, and uniform bounds for $g_{i}$ and its partial derivatives. By Lemma 10 in the Appendix there exists such a compact set in which the prices of profit-maximizing firms stay whatever the price-setting of the competitors.

With this result equilibrium existence is proved in a world with rational firms and consumers. The trade-off between the different kinds of heterogeneity as pointed out in Remarks 3 and 4 also holds in this case. I assumed that firms satisfy a minimal market share. This restriction on the strategy set can be avoided under a stronger assumption on $G_{i}$. Since the distribution function $G_{i}$ takes any value between 0 and 1 in order to show that profit function $\pi_{i}$ is $\log$-concave in $q_{i}$ it would be sufficient to show that

$$
\left(\frac{d^{2} D\left(q_{i}\right)}{d q_{i}^{2}}+\frac{d^{2} E\left(q_{i}\right)}{d q_{i}^{2}}\right)\left(D\left(q_{i}\right)+E\left(q_{i}\right)\right)-\left(\frac{d D\left(q_{i}\right)}{d q_{i}}+\frac{d E\left(q_{i}\right)}{d q_{i}}\right)^{2}<0
$$

This inequality holds for prices such that a firm has a negligible market share under the following assumption: 
- (A.9). $g_{i}$ is uniformly bounded from below on int $\Theta_{i}, i \in N$.

This assumption implies that $\partial G_{i}\left(\left(q_{j}-q_{i}\right)_{j \neq i}\right) / \partial q_{i}$ evaluated at $\theta_{i} \in\left\{\theta_{i} \in\right.$ $\left.\Theta_{i} \mid \exists j \neq i: \theta_{i j}=\underline{\theta}_{i j}\right\}$ is bounded away from zero and so is $d D\left(q_{i}\right) / d q_{i}$. For example, any truncated Normal distribution on $\Theta_{i}$ satisfies (A.9).

Under the additional assumption (A.9) equilibrium existence can be shown without the restriction of minimal market shares. I now state the main result of the paper. Like the previous results it formalizes the idea that aggregation reduces the price sensitivity of market expenditure and log-concave densities imply shape restrictions on the demand for each differentiated good so that profit functions are "well-behaved" on a sufficiently large set of prices. It is the last step to show that this idea is compatible with fully rational consumers and firms without the need to impose any restrictions on the firms' strategy spaces.

Theorem 4 Assume (A.1)-(A.5),(A.7),(A.9). There exists a pure-strategy BertrandNash equilibrium if $m_{2}$ is sufficiently small.

Sketch of Proof. Follows from the proofs of Lemmata 9 and 10, Theorem 3 and the argument above.

To summarize this section, I provide a table which presents the assumptions for the results in the different specifications.

\begin{tabular}{|l|l|}
\hline specification & assumptions \\
$\varepsilon$-maximizing firms (3.1) & (A.1)-(A.6), $m_{1}$ "small" \\
bounded rational consumers (3.2) & $\left(\right.$ A.1)-(A.5),(A.7), $m_{2}$ "small" \\
firms with minimal market share (3.3) & $\left(\right.$ A.1)-(A.5),(A.7),(A.8), $m_{2}$ "small" \\
rational consumers and firms (3.3) & (A.1)-(A.5),(A.7),(A.9), $m_{2}$ "small" \\
\hline
\end{tabular}

Table I: existence of equilibrium

\section{Further results with bounded rational consumers}

In this section I derive further results on existence, uniqueness, and monotone comparative statics in which I make use of the properties of market demand. I restrict 
the analysis to the simplest case in which uniqueness can be obtained, i.e. I consider the case in which consumers are bounded rational and make errors in the decision making as specified in subsection 3.2. For the specifications in subsection 3.3 the analysis is more complicated but possible along the same lines in order to show uniqueness.

\subsection{On equilibrium existence and uniqueness}

For more than two firms, I assume in addition that the distribution functions $G_{i}$ satisfy the weakly dominant diagonal property defined below. This implies that the associated game is quasi-supermodular (Proposition 1). Equilibrium existence can then be shown independent of the demand heterogeneity measured by $m_{2}$ (Proposition 2). Assuming that differences in marginal costs lie in the support of the distribution over the switching points guarantees that all firms are active and make profits in an equilibrium. The uniqueness of the equilibrium (Theorem 5) is then proved by showing that logarithmic payoff functions satisfy the dominant diagonal property. The game is dominance solvable.

It is convenient to write the model as the game $\Gamma=\left\{N, P,\left(\pi_{i}, i \in N\right)\right\}$ where $N$ is the finite set of firms, $\pi_{i}$ the profit function of firm $i$, and $P$ is the set of strategy profiles, which was defined as $\times_{i \in N}\left[c_{i}, z\right] \cap\left\{\left(p_{i}\right)_{i \in N} \mid \log p_{j}-\log p_{i} \leq \bar{\theta}_{i j}\right\}$. Denote $P_{i}\left(p_{-i}\right)$ the strategy set of firm $i$ given $p_{-i}$. When prices in $P$ are replaced by logarithmic prices the set is denoted by $Q$. The game with log-profits as payoff functions and logarithmic strategy sets is denoted by $\Gamma^{\prime}=\left\{N, Q,\left(\log \pi_{i}, i \in N\right)\right\}$. It will be shown that the model is a particular log-supermodular game.

The game $\Gamma^{\prime}$ is smooth supermodular if $Q$ is a complete lattice and $\log \pi_{i}$ is twice differentiable with $\partial^{2} \log \pi_{i}(p) / \partial \log p_{i} \partial \log p_{j} \geq 0$, for all $i, j \in N, j \neq i$, and $p \in P$ (Topkis' Characterization Theorem). The game $\Gamma^{\prime}$ exhibits strong strategic complementarity if $\partial^{2} \log \pi_{i} / \partial \log p_{i} \partial \log p_{j}>0$, for all $i, j \in N, j \neq i$ and $p \in P .{ }^{4}$

\footnotetext{
${ }^{4}$ The fruitfulness of the theory of supermodular games for economics has been recognized by Vives (1990) and Milgrom and Roberts (1990b). One of the standard examples are simple models of price competition with differentiated products. In the present framework strategy sets depend on the choices of the other players which does not create any problems here (for a definition and some results see e.g. Milgrom and Roberts, 1990a). For an interpretation of strategic complementarity
} 
In this section I introduce an additional assumption on the distribution of the $\theta_{i}, i \in N$. Recall that $h_{k j}^{i}$ is an element of the Hessian of $\log G_{i}$.

- (A.10). For all $i, j \in N, j \neq i, H_{i}$ has the following weakly dominant diagonal

$$
\left|h_{j j}^{i}\right| \geq \sum_{k \neq j} h_{k j}^{i}, i=1, \ldots, n-1
$$

By Lemma 1 it follows from (A.4) that $G_{i}$ log-concave, which implies that $h_{j j}^{i} \leq$ 0 . If the cross derivatives $h_{k j}^{i}>0$ at some point, then $h_{j j}^{i}$ needs to be sufficiently negative for the weakly dominant diagonal to hold. Note also that in the case of a duopoly the log-concavity of $G_{i}, n=1,2$, implies that (A.10) is trivially satisfied.

The first result says that under the additional assumption (A.10) the game $\Gamma$ is quasi-supermodular.

Proposition 1 Assume (A.1)-(A.4),(A.7),(A.10). The game $\Gamma^{\prime}$ is smooth supermodular on $P$.

Proof. I check the conditions for smooth supermodularity of $\Gamma^{\prime}$. Given $p_{-i}$, each price is chosen from a compact interval in $\Re_{+}$. By construction, $Q$ is a complete lattice. For $i \in N, \log \pi_{i}$ is twice continuously differentiable (by the assumption of differentiability and due to the construction of $Q$ ). Cross derivatives are

$$
\begin{aligned}
\frac{\partial^{2} \log \pi_{i}}{\partial \log p_{i} \partial \log p_{j}}(p) & \geq 0 \text { for } i, j \in N, j \neq i \\
\operatorname{as} \frac{\partial^{2} \log \pi_{i}}{\partial \log p_{i} \partial \log p_{j}}(p) & =\frac{\partial^{2} \log G_{i}}{\partial q_{i} \partial q_{j}}\left(\left(q_{j}-q_{i}\right)_{j \neq i}\right) \\
& =-\sum_{k \neq i} \frac{\partial^{2} \log G_{i}}{\partial q_{k} \partial q_{j}}\left(\left(q_{j}-q_{i}\right)_{j \neq i}\right) \\
& =-h_{j j}^{i}-\sum_{k \neq j} h_{k j}^{i} \geq 0
\end{aligned}
$$

see also Bulow, Geanakoplos and Klemperer (1985). 
by (A.10) and Lemma 11 in the Appendix which says that $G_{i}$ is $\log$-concave in $\log p_{j}$ on its support.

Note that quasi-supermodularity can be shown on any set which is a complete lattice and which is a subset of $\times_{i \in N}\left[c_{i}, \infty\right) \cap\left\{p \mid \log p_{j}-\log p_{i} \leq \bar{\theta}_{i j}\right\}$. Note also that neither (A.5) nor $m_{2}$ "small" are assumed. This implies that one can show equilibrium existence independent of the individual conditional demand functions in the population although profit functions are not necessarily quasi-concave (even on a compact set such as $P$ ) if one can find a compact set of strategy profiles. This is always possible.

Proposition 2 Assume (A.1)-(A.4),(A.7),(A.10). There exists a pure-strategy Bertrand-Nash equilibrium in the specification with bounded rational consumers.

The proof is delegated to the appendix and is based on Tarski's fixed point theorem. In the duopoly, the result is more general than Theorem 2. For more than two firms (A.10) is not implied by log-concavity of $g_{i}$ and the assumption on switching parameters (A.10) replaces assumptions on the heterogeneity of conditional demand functions. Along the same lines equilibrium existence can also be shown in specification 1 with bounded rational firms.

Below I establish the uniqueness of equilibrium. The following assumption says that marginal costs of the firms are not allowed to be too different.

- (A.11). $\log c_{j}-\log c_{i} \in\left(\underline{\theta}_{i j}, \bar{\theta}_{i j}\right), i, j \in N, i<j$.

(A.11) is weaker than (A.8). Under the assumption corner solutions can be excluded, i.e. all firms $i \in N$ will have positive market shares in equilibrium charging prices above there marginal costs and thus make profits.

Lemma 12 Assume (A.1)-(A.3),(A.11). If there is a pure-strategy Bertrand-Nash equilibrium all firms are active and make positive profits in equilibrium. 
Proof. Suppose that exactly one firm is inactive and show that this cannot be an equilibrium. The same argument applies for more than one inactive firm.

(i) Assume first that the inactive firm $i$ chooses a price $p_{i}$ above marginal costs such that the other firms together serve the whole market. They will choose their prices such that $\log p_{j}-\log p_{i}=\underline{\theta}_{i j}$ for all $j \neq i$. Such a price $p_{i}$ cannot be a best response for firm $i$ because by decreasing its price it can make positive profits.

(ii) Let firm $i$ choose its price equal to marginal costs so that the competitors can only serve the whole market by setting some of the prices below marginal costs. This cannot be a best response of all competitors. They will set their prices above marginal cost because they still can obtain some share of the market. In such a situation it cannot be optimal for firm $i$ to charge a price equal to marginal cost.

(iii) Clearly, firms do not set prices below marginal costs in equilibrium because at least one of these firms has a positive market share and thus makes losses.

Consequently, for $m_{2}$ sufficiently small every equilibrium price $p^{*} \in$ int $P$ and the analysis can be restricted to prices in $P$.

Lemma 13 says that if there is sufficient heterogeneity of demand behavior measured by small $m_{2}$, log-profits satisfy the dominant diagonal property. It is the last step to show uniqueness of the equilibrium. The proof is delegated to the appendix.

Lemma 13 Assume (A.1)-(A.5),(A.7),(A.10),(A.11). If $m_{2}$ sufficiently small, then log-profits satisfy the dominant diagonal property in log-prices on $Q$, i.e.

$$
-\frac{\partial^{2} \log \pi_{i}}{\left(\partial \log p_{i}\right)^{2}}(p)>\sum_{j \in N, j \neq i}\left|\frac{\partial^{2} \log \pi_{i}}{\partial \log p_{i} \partial \log p_{j}}(p)\right| \text { for } i, j \in N, j \neq i \text {. }
$$

If (A.10) is strengthened by making the left-hand side of the inequality by some $\epsilon$ greater than the right-hand side one can allow for less demand heterogeneity, i.e. $m_{2}$ can be greater. Proposition 1 and Lemma 13 put together give the uniqueness of equilibrium. A game is called dominance solvable if there exists only one serially undominated strategy profile. 
Theorem 5 Assume (A.1)-(A.5),(A.7),(A.10),(A.11) and $m_{2}$ sufficiently small. The pure strategy Bertrand-Nash equilibrium $p^{*}$ is unique. The original game $\Gamma$ is dominance solvable.

Proof. By Lemmata 8 and 12 there does not exist an equilibrium outside the interior of $P$. Furthermore, prices outside $P$ cannot belong to the set of serially undominated strategy profiles. In particular, one can use the proof of Lemma 8 in order to show that any price vector $p$ with some component $p_{i}>z, i \in N$, is serially dominated. On $P$ the dominant diagonal property holds. It implies that there exists a unique pure-strategy Nash equilibrium $p^{*}$ in the game $\Gamma^{\prime}$. Since, in addition, the game is smooth supermodular it is dominance solvable by Milgrom and Roberts (1990b, Theorem 5). Since serially undominated strategies are obtained by ordinal comparison and the transformation of the payoff functions is strictly increasing, the strategy profile $p^{*}$ is the unique serially undominated strategy profile of the game $\Gamma$ and, consequently, the unique pure-strategy Bertrand-Nash equilibrium.

It is well known that dominance solvability gives a strong prediction of play. In particular, only serially undominated strategies can be rationalizable (as defined by Bernheim, 1984) and only serially undominated strategies can be played with positive probability at a pure-strategy Nash equilibrium, mixed-strategy Nash equilibrium or correlated equilibrium (see Milgrom and Roberts, 1991, on rationality requirements for the firms to play the dominance solution see Tan and Werlang, 1988). Hence, $p^{*}$ is the unique rationalizable strategy profile and there are no other mixed-strategy Nash or correlated equilibria.

\subsection{Monotone comparative statics}

In the second part of this section I provide comparative statics results. Equilibrium prices are weakly increased as unit costs of one firm rise or as output taxes are increased. Under strong strategic complementarity they are strongly increased.

To obtain comparative statics results I look at a family of games. Let the payoff 
functions and strategy sets be parametrized by a parameter $\tau \in \Re^{K}$. Games in this family differ only in the value $\tau$ takes. Denote $Q_{i}\left(q_{-i}\right) \geq Q_{i}^{\prime}\left(q_{-i}\right)$ if the maximal and the minimal element of $Q_{i}\left(q_{-i}\right)$ is larger than or equal to the corresponding element in $Q_{i}^{\prime}\left(q_{-i}\right)$.

Suppose that $\Gamma^{\prime}(\tau)=\left\{N, Q(\tau),\left(\log \pi_{i}(\cdot, \tau), i \in N\right), \tau \in \Re^{K}\right\}$ is a family of smooth supermodular games satisfying $\partial^{2} \log \pi_{i} / \partial \log p_{i} \partial \tau_{k} \geq 0$ for all $i, k$ and let $Q_{i}\left(q_{-i}, \tau\right)$ be a compact interval in $\Re$. If $\log p^{*}(\tau)$ is the unique pure-strategy Nash equilibrium of the game then it is easy to show that $\tau_{k} \geq \tau_{k}^{\prime}$ for all $k$ and $Q_{i}\left(q_{-i}, \tau\right) \geq$ $Q_{i}\left(q_{-i}, \tau^{\prime}\right)$ for all $i$ imply that $p^{*}(\tau) \geq p^{*}\left(\tau^{\prime}\right)$ (by Theorem 5 in Milgrom and Roberts, 1990a, and Theorem 6 in combination with Theorem 5 in Milgrom and Roberts, 1990b, see also Cooper and John, 1988).

A family of games $\Gamma^{\prime}(\tau)$ exhibits multiplier effects in the parameter $\tau_{k}$ if the optimal reaction of each player is larger than its optimal reaction given the strategies of the other players, i.e. if $d \log p_{j}^{*} / d \tau_{k}>d \log p_{j}^{*} /\left.d \tau_{k}\right|_{p_{-i}} \geq 0$ for all firms and $>0$ for at least one firm. It is easy to show the following result: assume that a family of games $\Gamma(\tau)$ has a unique pure-strategy Nash equilibrium for each $\tau$, is smooth supermodular, exhibits strong strategic complementarity, and satisfies $\partial^{2} \log \pi_{i} / \partial \log p_{i} \partial \tau_{k} \geq 0$ for all $i, k$ with strict inequality for at least one firm and let all strategy sets be nondecreasing in $\tau$ then the family of games exhibits multiplier effects. The magnitude of the multiplier effect depends on the sensitivity of strategy choices to shocks and on the strength of strategic complementarities.

The first comparative statics result is obtained for changes in the marginal $\operatorname{cost} c_{j}$ of firm $j$. What happens to equilibrium prices $p^{*}$ when unit $\operatorname{cost} c_{j}$ is increased for some $j \in N$ ? I look at the family of games $\Gamma^{\prime}\left(c_{j}\right)=\left\{N, Q\left(c_{j}\right)\right.$, $\left.\left(\log \pi_{i}\left(p ; c_{j}\right), i \in N\right), c_{j} \in\left[\underline{c}_{j}, \bar{c}_{j}\right]\right\}$ where $Q_{i}\left(q_{-i}, c_{j}\right)$ is the strategy set of firm $i$. Under the assumptions of Theorem 5 (with (A.10) valid for the range of cost parameters under consideration), equilibrium prices $p^{*}$ are nondecreasing functions of unit cost $c_{j}$ of firm $j \in N$ because for $c_{m}>c_{m}^{\prime}, Q_{i}\left(q_{-i,} c_{j}\right) \geq Q_{i}\left(q_{-i}, c_{j}^{\prime}\right)$ for all $i \in N$ and

$$
\begin{aligned}
\frac{\partial^{2} \log \pi_{i}}{\partial \log p_{i} \partial c_{j}} & =0, i \neq j, \\
\frac{\partial^{2} \log \pi_{j}}{\partial \log p_{j} \partial c_{j}} & =\frac{p_{j}}{\left(p_{j}-c_{j}\right)^{2}}>0 .
\end{aligned}
$$


If, in addition, $H_{i}, i \in N$, has a strict dominant diagonal then the game $\Gamma^{\prime}$ exhibits strong strategic complementarities and the family of games exhibits multiplier effects: a cost shock to one single firm is transmitted into price increases of all firms in the market.

Suppose that the revenue of firm $i$ is taxed by a tax $t$ per unit of output sold. Profits of firm $i$ are

$$
\pi_{i}(p, t)=\frac{p_{i}-t-c_{i}}{p_{i}} B_{0}\left(p_{i}\right) G\left(\left(\log p_{j}-\log p_{i}\right)_{j \neq i}\right)
$$

For firm $i$ the tax simply is an additional cost. If assumption (A.10) is adjusted such that unit costs including tax satisfy (A.10) for $t \in[0, \bar{t}]$, equilibrium prices $p^{*}$ are nondecreasing functions of the tax per unit $t, t \in[0, \bar{t}]$. If one requires in addition the strict dominant diagonal of $H_{i}, i \in N$, then the family of games $\Gamma(t)$ exhibits multiplier effects. The result remains unchanged if firms face different taxes. Results are analogous in the case of revenue taxes.

If profits are taxed or the mass of consumers changes (i.e., multiplicative change), equilibrium prices are unaffected. An additive demand shock leads to monotone comparative statics but it is not clear what this shock means in terms of the variables at the individual level.

\section{Conclusion}

In models of imperfect competition the existence of equilibrium is far from guaranteed. Previous literature has looked at discrete choice models under particular functional form assumptions of individual demand. In this paper I replace functional form assumptions by heterogeneity of demand behavior. In particular, I show that aggregation along the lines of Grandmont is compatible with the discrete choice setup which is widely used in the theoretical and empirical literature. Future research has to show whether a more general version of aggregation can give rise to restrictions which are useful for the analysis of markets in which firms strategically interact. To summarize the main findings of this paper, aggregation of a heterogeneous population of consumers leads to quasi-concave profit functions on a compact set of prices and this property is used to show existence of equilibrium. 
Some modifications can be accommodated. As remarked in section 2, I analyzed a model of global competition in which each firm's price change has an effect on the demand of each competitor. The model is easily adjusted to allow for localized competition in which each firm only competes directly with a strict subset of the set of all competitors (this set can depend on the price vector $p$ ). For instance in a onedimensional model of product differentiation each firm has at most two neighbors (under a convexity assumption in the utility function, see e.g. Anderson, de Palma, and Thisse, 1992). In such a model one can index firms such that firm $i$ competes directly with firms $i-1$ and $i+1$ if all firms are active. Some assumptions on the costs of production imply that all firms are active in any candidate equilibrium. Consider the hypothetical profit function $\tilde{\pi}_{i}\left(p_{i-1}, p_{i}, p_{i+1}\right)$ of firm $i$ when it is ignoring the presence of all other firms other than its direct neighbors. If firm $i$ prices its good such that firm $i-1$ or firm $i+1$ is out of the market $\pi_{i}(p) \leq \tilde{\pi}_{i}\left(p_{i-1}, p_{i}, p_{i+1}\right)$. Hence, the critical part of the proof of equilibrium existence is the quasi-concavity of profit functions $\tilde{\pi}_{i}\left(p_{i-1}, p_{i}, p_{i+1}\right)$. Results are analogous to the ones derived in this paper (see also Peitz, 1998). In environments where vertical elements of product differentiation such as quality play a role one might want to allow for a correlation between $a, \beta$, and $\theta_{1}$. Such an extension is possible for particular specifications.

The model allows for unrestricted individual demand functions. Alternatively, one might want to work with shape restrictions on individual demand which are preserved under aggregation (this corresponds to assumptions on the third derivative of the utility function). This has been done by Dierker (1991, Proposition 6) who provides a more general condition on individual demand than unit elastic demand which is for instance satisfied by CES utility functions. If mean demand of each type $\theta_{1}$ is log-concave in logarithmic price on a set of consumers of measure 1, i.e. for a subset of $\Theta_{1}$ which is of full measure the price elasticity of the mean demand of type $\theta_{1}$ is nonincreasing, equilibrium existence can be shown without the heterogeneity assumptions ( $m_{1}$ or $m_{2}$ small) made in this paper.

In addition to the results on equilibrium existence, a sufficient condition for dominance solvability has been provided, which implies monotone comparative statics results. It should be pointed out that an initial price vector converges to the unique equilibrium price vector of the stage-game under a large class of learning rules (see Milgrom and Roberts, 1991). Suppose for example that learning is "slow", i.e. in 
period $t$ firms deviate with positive probability from the previous price $p_{i}^{t-1}$. If this deviation is the best response to $p_{-i}^{t-1}$, prices will converge to $p^{*}$ in a probabilistic sense as time goes to infinity (this result can be generalized). 


\section{Appendix}

Proof of Lemma 1. (see Dierker, 1991). Take $i=1$. Analogously for $i>1$. Define the convex sets

$$
\begin{aligned}
& C_{1}=\left\{\theta_{1} \mid \theta_{1 j} \leq \log p_{j}-\log p_{1}, \theta_{1 j} \in\left[\underline{\theta}_{1 j}, \bar{\theta}_{1 j}\right] \text { for all } j \in N, j \neq 1\right\}, \\
& \tilde{C}_{1}=\left\{\theta_{1} \mid \theta_{1 j} \leq \log p_{j}, \theta_{1 j} \in\left[\underline{\theta}_{1 j}, \bar{\theta}_{1 j}\right] \text { for all } j \in N, j \neq 1\right\} .
\end{aligned}
$$

Define $\tilde{g}_{1}\left(\theta_{12}, \ldots, \theta_{1 n}, \log p_{1}\right)=g_{1}\left(\theta_{12}+\log p_{1}, \ldots, \theta_{1 n}+\log p_{1}\right)$. Since $\log g_{1}$ is concave, so is $\log \tilde{g_{1}}$. One has

$$
\begin{aligned}
& \log G_{1}\left(\left(\log p_{j}-\log p_{1}\right)_{j \in N, j \neq 1}\right) \\
= & \int_{C_{1}} g_{1}\left(\theta_{1}\right) d \theta_{1} \\
= & \int_{\tilde{C}_{1}} g_{1}\left(\theta_{12}+\log p_{1}, \ldots, \theta_{1 n}+\log p_{1}\right) d \theta_{1} \\
= & \int_{\tilde{C}_{1}} \tilde{g}_{1}\left(\theta_{12}, \ldots, \theta_{1 n}, \log p_{1}\right) d \theta_{1}
\end{aligned}
$$

By Theorem 6 of Prékopa (1973) $G_{1}$ is a $\log$-concave function of $\log p_{1}$.

Lemma 4 Assume (A.1)-(A.4). Profits $\left(\left(p_{i}-c_{i}\right) / p_{i}\right) G_{i}\left(\left(\log p_{j}-\log p_{i}\right)_{j \neq i}\right)$ are quasi-concave in its own price.

Proof. Let me first show that profit is strictly log-concave in its logarithmic price where demand is strictly positive and price larger marginal cost. Consider firm 1. The proof goes through for all $i \in N$. Lemma 1 says that $G_{1}$ is $\log$-concave in $\log p_{1}$. $\log \left(p_{1}-c_{1}\right)-\log p_{1}$ is strictly concave in $\log p_{1}$ (see Remark 1 ).

Now look at quasi-concavity of profits for all cases. Quasi-concavity is violated if there exists a $p_{i 0}, p_{i 1}$, and $p_{i \lambda}$ with $c_{i} \leq p_{i 0}<p_{i 1}$ and $p_{i \lambda}=\lambda p_{i 0}+(1-\lambda) p_{i 1}, \lambda \in(0,1)$ such that

$$
\begin{aligned}
\left(p_{i 0}-c_{i}\right) G_{i}\left(\left(\log p_{j}-\log p_{i 0}\right)_{j \neq i}\right) & >\left(p_{i \lambda}-c_{i}\right) G_{i}\left(\left(\log p_{j}-\log p_{i \lambda}\right)_{j \neq i}\right) \\
\text { and }\left(p_{i 1}-c_{i}\right) G_{i}\left(\left(\log p_{j}-\log p_{i 1}\right)_{j \neq i}\right) & >\left(p_{i \lambda}-c_{i}\right) G_{i}\left(\left(\log p_{j}-\log p_{i \lambda}\right)_{j \neq i}\right)
\end{aligned}
$$


Two cases remain to be considered.

Case i) $p_{i 0}=c_{i}$. The first inequality requires that $p_{i \lambda}<c_{i}$ which is a contradiction. Case ii) $p_{i 0}>c_{i}$. Keep $p_{-i}$ fixed. For $G_{i}\left(\left(\log p_{j}-\log p_{i 1}\right)_{j \neq i}\right)=0$ the second inequality requires $p_{i \lambda}<c_{i}$ which is a contradiction. I already showed that profit is log-concave in its logarithmic price where demand is strictly positive and price larger marginal cost. As the logarithmic function is monotonic profits are quasi-concave.

Definition The best response correspondence $R$ is defined as

$$
\begin{aligned}
R: \quad \times_{i \in N}\left[c_{i}, z\right] \longrightarrow \times_{i \in N}\left[c_{i}, z\right] & \\
& \left(p_{1}, \ldots, p_{n}\right) \longmapsto\left(r_{1}\left(p_{-1}\right), \ldots, r_{n}\left(p_{-n}\right)\right) \equiv R(p)
\end{aligned}
$$

where

$$
r_{i}\left(p_{-i}\right)= \begin{cases}\left\{p_{i}^{+} \mid \pi\left(p_{i}^{+}, p_{-i}\right)=\max _{p_{i}}: \pi\left(p_{i}, p_{-i}\right)\right\} \cap\left[c_{i}, z\right] & \text { if } \neq \emptyset \\ \{z\} & \text { else. }\end{cases}
$$

Lemma 5 Let profit functions be quasi-concave in their own price. There exists a pure-strategy Bertrand-Nash equilibrium for $c_{i} \leq p_{i} \leq z$.

Proof. The best response correspondence $R$ is a correspondence with compact convex domain into itself. Since profit functions are continuous the best response correspondence is upper-hemicontinuous. The quasi-concavity of the profit functions guarantees that $R$ is convex-valued. Hence, one can make use of Kakutani's fixed point theorem which says that there exists a $p^{*}$ such that $p^{*} \in R\left(p^{*}\right)$.

Lemma 6 Assume (A.1). There is no $p_{i}>z, i \in N$, such that $\pi_{i}\left(p_{i}, p_{-i}^{*}\right)>$ $\pi_{i}\left(p_{i}^{*}, p_{-i}^{*}\right)$ where $\pi_{i}(p)=\left(\left(p_{i}-c_{i}\right) / p_{i}\right) G_{i}\left(\left(\log p_{j}-\log p_{i}\right)_{j \neq i}\right)$.

Proof. Suppose there is $p_{i}>z, i \in N$ such that $\pi_{i}\left(p_{i}, p_{-i}^{*}\right)>\pi_{i}\left(p_{i}^{*}, p_{-i}^{*}\right)$.

Case i). If there were a $j, j \neq i$, with $\log p_{j}^{*} \leq \log z+\underline{\theta}_{i j}$ then $\pi_{i}\left(p_{i}, p_{-i}^{*}\right)=0$ for all $p_{i}>z$ contradicting $\pi_{i}\left(p_{i}, p_{-i}^{*}\right)>\pi_{i}\left(p_{i}^{*}, p_{-i}^{*}\right)$.

Case ii). $\log z+\underline{\theta}_{i j} \leq \log p_{j}^{*}$ for all $j, j \neq i . p_{i}>z \geq p_{j}^{*}$ leads to a profit for firm $i$ of

$$
\frac{p_{i}-c_{i}}{p_{i}} G_{i}\left(\left(\log p_{j}^{*}-\log p_{i}\right)_{j \in N, j \neq i}\right) \leq \frac{p_{i}-c_{i}}{n p_{i}}
$$


because $G_{i}(0)=1 / n \geq G_{i}\left(\left(\log p_{j}^{*}-\log p_{i}\right)_{j \in N, j \neq i}\right)$. For $\log p_{i} \geq \log p_{j}^{*}-\underline{\theta}_{i j}$, for some $j$, one has $G_{i}\left(\left(\log p_{j}^{*}-\log p_{i}\right)_{j \in N, j \neq i}\right)=0$. Thus one must have, for all $j$, $\log p_{i}<\log p_{j}^{*}-\underline{\theta}_{i j}$ which is equivalent to $p_{i}<p_{j}^{*} / e^{\underline{\theta}_{i j}}$. Consequently, setting $p_{i}>z$ gives a payoff

$$
\begin{aligned}
\log \pi_{i}\left(p_{i}, p_{-i}^{*}\right) & =\log \frac{p_{i}-c_{i}}{p_{i}}+\log G_{i}\left(\left(\log p_{j}^{*}-\log p_{i}\right)_{j \in N, j \neq i}\right) \\
& <\log \left(1-\frac{c_{i}}{p_{k}^{*}} e^{\theta_{i j}}\right)-\log n
\end{aligned}
$$

for all $j$. Set $\log p_{i}^{\prime}=\min \left\{\left(\log p_{j}^{*}-\bar{\theta}_{i j}\right)_{j}\right\}$ and denote the index of the minimal element with $k$. Profit of firm $i$ setting $p_{i}^{\prime}$ is

$$
\log \pi_{i}\left(p_{i}^{\prime}, p_{-i}^{*}\right)=\log \left(\frac{p_{k}^{*}}{e^{\bar{\theta}_{i k}}}-c_{i}\right)-\log \frac{p_{k}^{*}}{e^{\bar{\theta}_{i k}}} .
$$

It is not in the interest of the firm to set $p_{i}>z \geq p_{k}^{*}$ if $\pi_{i}\left(p_{i}, p_{-i}^{*}\right)<\pi_{i}\left(p_{i}^{\prime}, p_{-i}^{*}\right)$.

$$
\begin{aligned}
& \Longleftarrow \log \left(1-\frac{c_{i}}{p_{k}^{*}} e^{\theta_{i k}}\right)-\log n<\log \left(1-\frac{c_{i}}{p_{k}^{*}} e^{\bar{\theta}_{i k}}\right) \\
& \Longleftarrow e^{-\underline{\theta}_{i k}} p_{k}^{*}>\frac{n}{n-1} c_{i}\left(e^{\bar{\theta}_{i k}}-e^{\underline{\theta}_{i k}}\right) \\
& \Longleftarrow \log p_{k}^{*}>\log z+\underline{\theta}_{i k}
\end{aligned}
$$

Since this inequality is satisfied, a deviation from $p_{i}^{*}$ is not profitable and one has $\pi_{i}\left(p_{i}, p_{-i}^{*}\right)<\pi_{i}\left(p_{i}^{\prime}, p_{-i}^{*}\right) \leq \pi_{i}\left(p_{i}^{*}, p_{-i}^{*}\right)$ for $p_{i}>z$. This holds for all $i \in N$.

Lemma 8 Assume (A.1)-(A.3),(A.5). For $m_{2}$ sufficiently small, there is no $p_{i}>z$, $i \in N$, such that $\pi_{i}\left(p_{i}, p_{-i}^{*}\right)>\pi_{i}\left(p_{i}^{*}, p_{-i}^{*}\right)$ in the specification with bounded rational consumers.

Proof. Suppose there is $p_{i}>z, i \in N$ such that $\pi_{i}\left(p_{i}, p_{-i}^{*}\right)>\pi_{i}\left(p_{i}^{*}, p_{-i}^{*}\right)$.

Case i). As in the proof of Lemma 6.

Case ii). Part (i) following the proof of Lemma $6 . \log z+\underline{\theta}_{i j} \leq \log p_{j}^{*}$ for all $j, j \neq i$. $p_{i}>z \geq p_{j}^{*}$ leads to a profit for firm $i$ of

$$
\left(p_{i}-c_{i}\right) \frac{B_{0}\left(p_{i}\right)}{p_{i}} G_{i}\left(\left(\log p_{j}^{*}-\log p_{i}\right)_{j \in N, j \neq i}\right) \leq\left(p_{i}-c_{i}\right) \frac{B_{0}\left(p_{i}\right)}{n p_{i}}
$$


because $1 / n \geq G_{i}\left(\left(\log p_{j}^{*}-\log p_{i}\right)_{j \in N, j \neq i}\right)$. As in the proof of Lemma 6 one must have, for all $j, \log p_{i}<\log p_{j}^{*}-\underline{\theta}_{i j}$ which is equivalent to $p_{i}<p_{j}^{*} / e^{\theta_{i j}}$. Consequently, setting $p_{i}>z$ gives a payoff

$$
\begin{aligned}
\log \pi_{i}\left(p_{i}, p_{-i}^{*}\right) & =\log \frac{p_{i}-c_{i}}{p_{i}}+\log B_{0}\left(p_{i}\right)+\log G_{i}\left(\left(\log p_{j}^{*}-\log p_{i}\right)_{j \in N, j \neq i}\right) \\
& <\log \left(1-\frac{c_{i}}{p_{k}^{*}} e^{\Theta_{i j}}\right)+\log B_{0}\left(p_{i}\right)-\log n
\end{aligned}
$$

for all $j$. Set $\log p_{i}^{\prime}=\min \left\{\left(\log p_{j}^{*}-\bar{\theta}_{i j}\right)_{j}\right\}$ and denote the index of the minimal element with $k$. Profit of firm $i$ setting $p_{i}^{\prime}$ is

$$
\log \pi_{i}\left(p_{i}^{\prime}, p_{-i}^{*}\right)=\log \left(1-\frac{c_{i}}{p_{k}^{*}} e^{\bar{\theta}_{i k}}\right)+\log B_{0}\left(p_{i}^{\prime}\right) .
$$

It is not in the interest of the firm to set $p_{i}>z \geq p_{k}^{*}$ if $\pi_{i}\left(p_{i}, p_{-i}^{*}\right)<\pi_{i}\left(p_{i}^{\prime}, p_{-i}^{*}\right)$.

$$
\begin{gathered}
\Longleftarrow \log \left(1-\frac{c_{i}}{p_{k}^{*}} e^{\underline{\theta}_{i k}}\right)+\log B_{0}\left(p_{i}\right)-\log n \\
<\log \left(1-\frac{c_{i}}{p_{k}^{*}} e^{\bar{\theta}_{i k}}\right)+\log B_{0}\left(p_{i}^{\prime}\right)
\end{gathered}
$$

Part (ii). First I show that $\left|\log B_{0}\left(p_{i}\right)-\log B_{0}\left(p_{i}^{\prime}\right)\right|$ is small.

$$
\left|\log B_{0}\left(p_{i}\right)-\log B_{0}\left(p_{i}^{\prime}\right)\right| \leq \int_{\log p_{i}^{\prime}}^{\log p_{i}}\left|\frac{\partial \log B_{0}\left(p_{i}\right)}{\partial \log \widetilde{p}_{i}}\right| d \log \widetilde{p}_{i} \leq\left(\log p_{i}-\log p_{i}^{\prime}\right) \frac{m_{2}}{W}
$$

¿From the definition of $p_{i}$ and $p_{i}^{\prime}$ it follows that $\log p_{i}-\log p_{i}^{\prime} \leq \bar{\theta}_{i j}-2 \underline{\theta}_{i j}$. Hence, for any $\epsilon>0$ one can find an $m_{2}$ sufficiently small such that $\left|\log B_{0}\left(p_{i}\right)-\log B_{0}\left(p_{i}^{\prime}\right)\right|<$ $\log (1+\epsilon)$. It remains to be shown that

$$
\begin{gathered}
\log \left(1-\frac{c_{i}}{p_{k}^{*}} e^{\theta_{i k}}\right)+\log (1+\epsilon)<\log \left(1-\frac{c_{i}}{p_{k}^{*}} e^{\bar{\theta}_{i k}}\right)+\log n \\
\Longleftrightarrow p_{k}^{*}>\frac{1}{n-1-\epsilon} c_{i}\left(n e^{\bar{\theta}_{i k}}-(1+\epsilon) e^{\theta_{i k}}\right) \\
\Longleftarrow \log p_{k}^{*}>\log z+\underline{\theta}_{i k}
\end{gathered}
$$

for $m_{2}$ sufficiently small. (From the proof of Lemma 6 it is clear that at two different steps there is room for eliminating $\epsilon$.) In summary, one has $\pi_{i}\left(p_{i}, p_{-i}^{*}\right)<\pi_{i}\left(p_{i}^{\prime}, p_{-i}^{*}\right) \leq$ 
$\pi_{i}\left(p_{i}^{*}, p_{-i}^{*}\right)$ for $p_{i}>z$. This holds for all $i \in N$.

\section{Proof of Remark 5.}

$$
\log G(\tilde{\theta})=\log \int_{\underline{\theta}}^{\tilde{\theta}} e^{h(\theta)} d \theta \text { where } h(\theta)=\log g_{1}(\theta)
$$

It has to be shown that $d^{2} \log G(\tilde{\theta}) / d \tilde{\theta}^{2} \leq-\tilde{\kappa}$ which is implied by

$$
e^{h(\tilde{\theta})}\left(h^{\prime}(\tilde{\theta}) \int_{\underline{\theta}}^{\tilde{\theta}} e^{h(\theta)} d \theta-e^{h(\tilde{\theta})}\right) \leq-\tilde{\kappa}
$$

As $h$ is concave: $h^{\prime}(\tilde{\theta}) \leq h^{\prime}(\theta), \theta \in[\underline{\theta}, \tilde{\theta}]$.

$$
h^{\prime}(\tilde{\theta}) \int_{\underline{\theta}}^{\tilde{\theta}} e^{h(\theta)} d \theta \leq \int_{\underline{\theta}}^{\tilde{\theta}} h^{\prime}(\theta) e^{h(\theta)} d \theta=e^{h(\tilde{\theta})}-e^{h(\underline{\theta})} .
$$

Hence, $-e^{h(\tilde{\theta})} e^{h(\underline{\theta})}=-g_{1}(\tilde{\theta}) g_{1}(\underline{\theta}) \leq-\tilde{\kappa}$ because $g_{1}$ is bounded from below on its support.

Lemma 9 Assume (A.1)-(A.5), (A.7). The third additive term on the right-hand side of equation (3) can be bounded in absolute value by any positive number for $m_{2}$ sufficiently small.

Proof. (i) As shown in the text, the third additive term on the right-hand side of equation (3) can be made arbitrarily small in absolute value if for any $\epsilon$ one can find an $m_{2}$ such that inequality (5) is satisfied. Since firms have to satisfy a minimal market share $D\left(q_{i}\right) \geq \gamma$. This implies that $\epsilon\left(D\left(q_{i}\right)+E\left(q_{i}\right)\right)^{2}>\epsilon\left(D\left(q_{i}\right)-\left|E\left(q_{i}\right)\right|\right)^{2}>$ $\widetilde{\epsilon}>0$ if $\left|E\left(q_{i}\right)\right|$ can be made arbitrarily small.

(ii) $\left|E\left(q_{i}\right)\right|$ can be made arbitrarily small: for any positive number one can find a value for $m_{2}$ sufficiently small such that $\left|E\left(q_{i}\right)\right|$ is less than this number.

$$
\left|E\left(q_{i}\right)\right|=\left|\int \frac{B\left(p_{i}, \theta_{i 1}\right)-B_{0}\left(p_{i}\right)}{B_{0}\left(p_{i}\right)} g_{i}\left(\theta_{i}\right) d \theta_{i}\right|
$$




$$
\begin{aligned}
& \leq \frac{1}{\delta W} \int\left|B\left(p_{i}, \theta_{i 1}\right)-B_{0}\left(p_{i}\right)\right| g_{i}\left(\theta_{i}\right) d \theta_{i} \\
& \leq \frac{1}{\delta W} \int\left|\theta_{i 1}\right| m_{2} W g_{i}\left(\theta_{i}\right) d \theta_{i}(\text { by Lemma } 3) \\
& \leq \frac{m_{2}}{\delta}\left(\bar{\theta}_{i 1}-\underline{\theta}_{i 1}\right) D\left(q_{i}\right)
\end{aligned}
$$

Hence, $\epsilon\left(D\left(q_{i}\right)-\left|E\left(q_{i}\right)\right|\right)^{2} \geq \epsilon\left(1-\frac{m_{2}}{\delta}\left(\bar{\theta}_{i 1}-\underline{\theta}_{i 1}\right)\right)^{2} \gamma^{2}>\widetilde{\epsilon}$ for $m_{2}$ sufficiently small.

(iii) It remains to be shown that the left-hand side of inequality (5) can be made arbitrarily small in absolute value.

$$
\begin{aligned}
& \left|\frac{d^{2} E\left(q_{i}\right)}{d q_{i}^{2}}\left(D\left(q_{i}\right)+E\left(q_{i}\right)\right)+\frac{d^{2} D\left(q_{i}\right)}{d q_{i}^{2}} E\left(q_{i}\right)-2 \frac{d D\left(q_{i}\right)}{d q_{i}} \frac{d E\left(q_{i}\right)}{d q_{i}}-\left(\frac{d E\left(q_{i}\right)}{d q_{i}}\right)^{2}\right| \\
\leq & \left|\frac{d^{2} E\left(q_{i}\right)}{d q_{i}^{2}}\right|\left(D\left(q_{i}\right)+\left|E\left(q_{i}\right)\right|\right)+\left|\frac{d^{2} D\left(q_{i}\right)}{d q_{i}^{2}}\right|\left|E\left(q_{i}\right)\right|+2\left|\frac{d D\left(q_{i}\right)}{d q_{i}}\right|\left|\frac{d E\left(q_{i}\right)}{d q_{i}}\right|+\left(\frac{d E\left(q_{i}\right)}{d q_{i}}\right)^{2}
\end{aligned}
$$

Remark that $D\left(q_{i}\right) \leq 1$. By (A.1) $g_{i}$ and its partial derivatives are uniformly bounded from above. This implies that $\left|d D\left(q_{i}\right) / d q_{i}\right|$ and $\left|d^{2} D\left(q_{i}\right) / d q_{i}^{2}\right|$ are uniformly bounded from above. It remains to be shown that $\left|E\left(q_{i}\right)\right|,\left|d E\left(q_{i}\right) / d q_{i}\right|$, and $\left|d^{2} E\left(q_{i}\right) / d q_{i}^{2}\right|$ can be made arbitrarily small. From part (ii) it follows that $\left|E\left(q_{i}\right)\right|$ can be made arbitrarily small.

(iv) $\left|d E\left(q_{i}\right) / d q_{i}\right|$ can be made arbitrarily small:

$$
\begin{aligned}
\frac{d E\left(q_{i}\right)}{d q_{i}}= & \frac{d}{d q_{i}}\left(\int_{\times_{j \in N, j \neq i}\left[\underline{\theta}_{i j}, q_{j}-q_{i}\right]} \frac{B\left(e^{q_{i}}, \theta_{i 1}\right)-B_{0}\left(e^{q_{i}}\right)}{B_{0}\left(e^{q_{i}}\right)} g_{i}\left(\theta_{i}\right) d \theta_{i}\right) \\
= & \int_{\times_{j \in N, j \neq i}\left[\underline{\theta}_{i j}, q_{j}-q_{i}\right]} \frac{d\left(\left(B\left(e^{q_{i}}, \theta_{i 1}\right)-B_{0}\left(e^{q_{i}}\right)\right) / B_{0}\left(e^{q_{i}}\right)\right)}{d q_{i}} g_{i}\left(\theta_{i}\right) d \theta_{i} \\
& -\sum_{j \neq i} \int_{\times_{k \in N, k \neq i, k \neq j}\left[\underline{\theta}_{i k}, q_{k}-q_{i}\right]}^{\frac{B\left(e^{q_{i}}, \theta_{i 1}\right)-B_{0}\left(e^{q_{i}}\right)}{B_{0}\left(e^{q_{i}}\right)}} \\
& g_{i}\left(\theta_{i 1}, \ldots, \theta_{i, j-1}, q_{j}-q_{i}, \theta_{i, j+1}, \ldots, \theta_{i n}\right) d \theta_{i 1} \cdots d \theta_{i, j-1} d \theta_{i, j+1} \cdots d \theta_{i n}
\end{aligned}
$$

As shown in part (ii) of the proof $\left|\left(B\left(p_{i}, \theta_{i 1}\right)-B_{0}\left(p_{i}\right)\right) / B_{0}\left(p_{i}\right)\right|$ can be made arbitrarily small. Since by (A.1) $g_{i}$ is uniformly bounded from above it remains to be shown that the first additive term can be made arbitrarily small in absolute value. 


$$
\begin{aligned}
& \left|\frac{d\left(\left(B\left(p_{i}, \theta_{i 1}\right)-B_{0}\left(p_{i}\right)\right) / B_{0}\left(p_{i}\right)\right)}{d \log p_{i}}\right| \\
= & \left|\frac{1}{B_{0}\left(p_{i}\right)} \frac{d B\left(p_{i}, \theta_{i 1}\right)}{d \log p_{i}}-\frac{B\left(p_{i}, \theta_{i 1}\right)}{B_{0}\left(p_{i}\right)} \frac{d \log B_{0}\left(p_{i}\right)}{d \log p_{i}}\right| \\
\leq & \frac{1}{\delta W}\left|\frac{d B\left(p_{i}, \theta_{i 1}\right)}{d \log p_{i}}\right|+\frac{W}{\delta W} \frac{m_{2}}{\delta}
\end{aligned}
$$

With the same argument as for $d B_{0}\left(p_{i}\right) / d \log p_{i}$ in the proof of Lemma 7 also $d B\left(p_{i}, \theta_{i 1}\right) / d \log p_{i}$ can be bounded in absolute value by $m_{2} W$. Consequently,

$$
\left|\frac{d\left(\left(B\left(p_{i}, \theta_{i 1}\right)-B_{0}\left(p_{i}\right)\right) / B_{0}\left(p_{i}\right)\right)}{d \log p_{i}}\right| \leq \frac{m_{2}}{\delta}\left(1+\frac{m_{2}}{\delta}\right),
$$

which can be made arbitrarily small for $m_{2}$ sufficiently small.

(v) Finally, also $\left|d^{2} E\left(q_{i}\right) / d q_{i}^{2}\right|$ can be made arbitrarily small. The analysis is lengthy and easily carried out along the lines of part (iv) above. Note that by (A.1) $g_{i}$ and its partial derivatives are uniformly bounded in absolute value. With the bounds provided in parts (ii) and (iv), it only remains to be shown that $d^{2}\left(\left(B\left(p_{i}, \theta_{i 1}\right)-B_{0}\left(p_{i}\right)\right) / B_{0}\left(p_{i}\right)\right) / d\left(\log p_{i}\right)^{2}$ can be made arbitrarily small in absolute value.

$$
\begin{aligned}
& \mid \frac{d^{2} \frac{\left(B\left(p_{i}, \theta_{i 1}\right)-B_{0}\left(p_{i}\right)\right)}{B_{0}\left(p_{i}\right)}\left|\leq \frac{1}{d\left(\log p_{i}\right)^{2}}\right| \leq}{B_{0}\left(p_{i}\right)}\left(B\left(p_{i}, \theta_{i 1}\right)\left(\left(\frac{d \log B_{0}\left(p_{i}\right)}{d \log p_{i}}\right)^{2}+\left|\frac{d^{2} \log B_{0}\left(p_{i}\right)}{d\left(\log p_{i}\right)^{2}}\right|\right)\right. \\
&\left.+2\left|\frac{d B\left(p_{i}, \theta_{i 1}\right)}{d \log p_{i}}\right|\left|\frac{d \log B_{0}\left(p_{i}\right)}{d \log p_{i}}\right|+\left|\frac{d^{2} B\left(p_{i}, \theta_{i 1}\right)}{d\left(\log p_{i}\right)^{2}}\right|\right)
\end{aligned}
$$

Each of the additive terms in parentheses can be made arbitrarily small and the result follows.

Lemma 10 Assume (A.1)-(A.3),(A.5). For $m_{2}$ sufficiently small, there is no $p_{i}>z$, $i \in N$, such that $\pi_{i}\left(p_{i}, p_{-i}^{*}\right)>\pi_{i}\left(p_{i}^{*}, p_{-i}^{*}\right)$ in the specifications of subsection 3.3.

Proof. I follow the proof of Lemma 8 with profit functions in equation (3) using the same definitions of prices $p_{i}$ and $p_{i}^{\prime}$. It only has to be shown that $\log \pi_{i}\left(p_{i}, p_{-i}^{*}\right)<$ $\log \pi_{i}\left(p_{i}^{\prime}, p_{-i}^{*}\right) . E\left(\log p_{i}\right)$ is defined as in the main text. 


$$
\begin{aligned}
& \log \left(1-\frac{c_{i}}{p_{k}^{*}} e^{\underline{\theta}_{i j}}\right)+\log B_{0}\left(p_{i}\right)+\log \left(\frac{1}{n}+E\left(\log p_{i}\right)\right) \\
< & \log \left(1-\frac{c_{i}}{p_{k}^{*}} e^{\bar{\theta}_{i k}}\right)+\log B_{0}\left(p_{i}^{\prime}\right)+\log \left(1+E\left(\log p_{i}^{\prime}\right)\right)
\end{aligned}
$$

As shown in part (ii) of the proof of Lemma $8\left|\log B_{0}\left(p_{i}\right)-\log B_{0}\left(p_{i}^{\prime}\right)\right|$ can be made arbitrarily small. As shown in part (ii) of the proof of Lemma $9\left|E\left(\log p_{i}\right)\right|$ can be made arbitrarily small for any $p_{i}$. Consequently, for any $\epsilon^{\prime}$ one can find an $m_{2}$ sufficiently small such that

$$
\left|\log B_{0}\left(p_{i}\right)-\log B_{0}\left(p_{i}^{\prime}\right)\right|-\log \left(1-\left|E\left(\log p_{i}^{\prime}\right)\right|\right)+\log \left(1+n\left|E\left(\log p_{i}\right)\right|\right)<\epsilon^{\prime} .
$$

Consequently, it remains to be shown that

$$
\log \left(1-\frac{c_{i}}{p_{k}^{*}} e^{\underline{\theta}_{i k}}\right)+\log \left(1+\epsilon^{\prime}\right)<\log \left(1-\frac{c_{i}}{p_{k}^{*}} e^{\bar{\theta}_{i k}}\right)+\log n
$$

which follows from the proof of Lemma 8 where $\epsilon$ has to be replaced by $\epsilon^{\prime}$.

Lemma 11 Assume (A.1),(A.4). $G_{i}\left(\left(\log p_{j}-\log p_{i}\right)_{j \neq i}\right)$ is log-concave in $\log p_{j}$, $j \neq i$.

Proof. (following Proposition 5 by Dierker, 1991, for $n$ firms). Take $i=1$. Analogously for $i>1$. Take $j=2$. Analogously for $i \neq 1, j \neq i$. Define the convex set

$$
\begin{gathered}
\breve{C}_{12}=\left\{\theta_{1} \mid \theta_{12} \leq-\log p_{1}, \theta_{1 k} \leq \log p_{k}-\log p_{1}, \text { for } k \geq 3,\right. \\
\left.\theta_{1 j} \in\left[\underline{\theta}_{1 j}, \bar{\theta}_{1 j}\right] \text { for all } j \in N, j \neq 1\right\} .
\end{gathered}
$$

Define $\breve{g}_{12}\left(\theta_{12}, \ldots, \theta_{1 n}, \log p_{2}\right)=g_{1}\left(\theta_{12}-\log p_{2}, \theta_{13}, \ldots, \theta_{1 n}\right)$. Since $\log g_{1}$ is concave in $\log p_{2}$, so is $\log \breve{g}_{12}$. One has

$$
\begin{aligned}
& \log G_{1}\left(\left(\log p_{j}-\log p_{1}\right)_{j \in N, j \neq 1}\right) \\
= & \int_{C_{1}} g_{1}\left(\theta_{1}\right) d \theta_{1}
\end{aligned}
$$




$$
\begin{aligned}
& =\int_{\breve{C}_{12}} g_{1}\left(\theta_{12}-\log p_{2}, \theta_{13}, \ldots, \theta_{1 n}\right) d \theta_{1} \\
& =\int_{\breve{C}_{12}} \breve{g}_{12}\left(\theta_{12}, \ldots, \theta_{1 n}, \log p_{2}\right) d \theta_{1}
\end{aligned}
$$

By Theorem 6 of Prékopa (1973) $G_{1}$ is a $\log$-concave function of $\log p_{2}$.

Proof of Proposition 2. (i) By Tarski's fixed point theorem and Proposition 1 there exists a fixed point in $\widetilde{\Gamma}=\left\{N, \widetilde{Q},\left(\log \pi_{i}, i \in N\right)\right\}$ where $\widetilde{Q}$ is a complete lattice which is a subset of $\times_{i \in N}\left[\log c_{i}, \infty\right) \cap\left\{\log p \mid \log p_{j}-\log p_{i} \leq \bar{\theta}_{i j}\right\}$. $\widetilde{P}$ is defined accordingly. Such a fixed point is an equilibrium on $\widetilde{P}$. It remains to be shown that one can construct a set $\widetilde{P}$ such that an equilibrium given the restricted set of strategy profiles $\widetilde{P}$ is also an equilibrium given the set of strategy profiles $\Re_{++}^{n}$.

(ii) Denote $\Delta^{\max }=\max \left\{\left(\bar{\theta}_{i j}-\underline{\theta}_{i j}\right)_{i, j}\right\}$. Note that $B_{0}\left(p_{i}\right) \leq W$. For $p_{i} \geq \bar{p}_{i}$, denote $\bar{B}_{0}\left(p_{i} ; \bar{p}_{i}\right)=\max _{\widehat{p}_{i} \in\left[\bar{p}_{i}, p_{i}\right]} B_{0}\left(\widehat{p}_{i}\right)$. By construction, the function $\bar{B}_{0}\left(\cdot ; \bar{p}_{i}\right)$ is nondecreasing in $p_{i}$. Hence, $\bar{B}_{0}\left(\cdot ; \bar{p}_{i}\right)$ converges to some value in $[0, W]$ as $p_{i}$ turns to infinity for any value of $\bar{p}_{i}$. This limit is nonincreasing in $\bar{p}_{i}$. Hence, given $\bar{B}_{0}$ for any $\epsilon>0$ there exist prices $\widetilde{p}_{i}$ such that $\log \bar{B}_{0}\left(p_{i} ; \widetilde{p}_{i}\right)-\log \bar{B}_{0}\left(\widetilde{p}_{i} ; \widetilde{p}_{i}\right)<\log (1+\epsilon)$ for any $p_{i} \in\left[\widetilde{p}_{i}, \widetilde{p}_{i}+\Delta^{\max }-\underline{\theta}_{i j}\right](\forall i \in N \exists j \neq i)$. Denote the sets of these prices $\widetilde{p}_{i}$ by $P^{+}(\epsilon)$. Fix for the moment $\epsilon$. Denote $z^{\prime}=\widetilde{p}_{i}+\Delta^{\max }$. The set $P^{+}(\epsilon)$ is a union of intervals and is unbounded. For some $\widetilde{p}_{i} \in P^{+}(\epsilon)$ construct compact strategy sets $\widetilde{P}=\times_{i \in N}\left[c_{i}, z^{\prime}\right) \cap\left\{\left(p_{i}\right)_{i \in N} \mid \log p_{j}-\log p_{i} \leq \bar{\theta}_{i j}\right\}$. Denote $\widetilde{P}_{i}\left(p_{-i}\right)=$ $\left\{p_{i} \mid\left(p_{i}, p_{-i}\right) \in \widetilde{P}\right\}$. The result is shown if firms do not set prices outside the set $\widetilde{P}_{i}\left(p_{-i}^{*}\right)$. Only prices $p_{i}$ with $z^{\prime}-\underline{\theta}_{i j} \geq p_{i} \geq z^{\prime}$ have to be considered because other prices outside $\widetilde{P}_{i}\left(p_{-i}^{*}\right)$ lead to zero or negative profits. If there is an equilibrium in $\widetilde{P}$ with $\log p_{j}^{*} \leq \log z^{\prime}+\underline{\theta}_{i j}$ for some $j \neq i$ then $\pi_{i}\left(p_{i}, p_{-i}^{*}\right)=0$ for all $p_{i}>z^{\prime}$ contradicting $\pi_{i}\left(p_{i}, p_{-i}^{*}\right) \geq \pi_{i}\left(p_{i}^{*}, p_{-i}^{*}\right)$. Suppose $\log p_{j}^{*}>\log z^{\prime}+\underline{\theta}_{i j}$ for all $j \neq i$. Clearly, at a price $\widetilde{p}_{i}, G_{i}\left(\left(\log p_{j}^{*}-\log \widetilde{p}_{i}\right)_{j \neq i}\right)=1$. Since $p^{*}$ is an equilibrium price vector in $\widetilde{P}$, one has

$$
\pi_{i}\left(p^{*}\right) \geq \pi_{i}\left(\widetilde{p}_{i}, p_{-i}^{*}\right)=\left(1-\frac{c_{i}}{\widetilde{p}_{i}}\right) B_{0}\left(\widetilde{p}_{i}\right) .
$$

A profit maximizing deviation has to be a price $p_{i}$ such that $z^{\prime}-\underline{\theta}_{i j} \geq p_{i} \geq z^{\prime}$ for 
all $j \neq i$. This gives profits

$$
\begin{aligned}
\pi_{i}\left(p_{i}, p_{-i}^{*}\right) & \leq\left(1-\frac{c_{i}}{p_{i}}\right) B_{0}\left(p_{i}\right) \frac{1}{n} \\
& \leq\left(1-\frac{c_{i}}{z^{\prime}-\underline{\theta}_{i j}}\right) B_{0}\left(p_{i}\right) \frac{1}{n}
\end{aligned}
$$

It can now be shown that such a deviation is not profitable for $\epsilon>0$ sufficiently small and $\widetilde{p}_{i} \in P^{+}(\epsilon)$ sufficiently large.

$$
\begin{gathered}
\log \left(1-\frac{c_{i}}{\widetilde{p}_{i}}\right)+\log B_{0}\left(\widetilde{p}_{i}\right) \geq \log \left(1-\frac{c_{i}}{\widetilde{p}_{i}+\Delta^{\max }-\underline{\theta}_{i j}}\right)+\log B_{0}\left(p_{i}\right)-\log n \\
\Longleftarrow \log n \geq \log \left(1-\frac{c_{i}}{\widetilde{p}_{i}+\Delta^{\max }-\underline{\theta}_{i j}}\right)-\log \left(1-\frac{c_{i}}{\widetilde{p}_{i}}\right)+\log (1+\epsilon)
\end{gathered}
$$

because $B_{0}\left(\widetilde{p}_{i}\right)=\bar{B}_{0}\left(\widetilde{p}_{i} ; \widetilde{p}_{i}\right)$ and $B_{0}\left(p_{i}\right) \leq \bar{B}_{0}\left(p_{i} ; \widetilde{p}_{i}\right)$.

Proof of Lemma 13. Remark that

$$
\frac{\partial^{2} \log \pi_{i}}{\partial \log p_{i} \partial \log p_{j}}=\frac{\partial^{2} \log G_{i}}{\partial q_{i} \partial q_{j}}\left(\left(q_{j}-q_{i}\right)_{j \in N, j \neq i}\right) \geq 0
$$

because of Proposition 1. Remark also that

$$
\frac{\partial^{2} \log G_{i}}{\left(\partial q_{i}\right)^{2}}=-\sum_{j \in N, j \neq i} \frac{\partial^{2} \log G_{i}}{\partial q_{i} \partial q_{j}} .
$$

It remains to be shown that

$$
\begin{array}{r}
-\frac{\partial^{2} \log \pi_{i}}{\left(\partial \log p_{i}\right)^{2}}>-\frac{\partial^{2} \log G_{i}}{\left(\partial \log p_{i}\right)^{2}} \\
\Longleftrightarrow \frac{p_{i} c_{i}}{\left(p_{i}-c_{i}\right)^{2}}-\frac{d^{2} \log B_{i}\left(p_{i}\right)}{\left(d \log p_{i}\right)^{2}}>0 \text { for } i \in N .
\end{array}
$$

These inequalities are satisfied if $m_{2}$ is sufficiently small, i.e. for all $i \in N, m_{2}$ satisfies

$$
\frac{m_{2}}{\delta}\left(1+\frac{m_{2}}{\delta}\right)<\frac{e^{z} c_{i}}{\left(e^{z}-c_{i}\right)^{2}}
$$




\section{References}

[1] S.P. Anderson, A. de Palma, and J.-F. Thisse (1992), "Discrete Choice Theory of Product Differentiation", MIT Press, Cambridge, Mass., 1992.

[2] M. Baye and J. Morgan (1996), Necessary and sufficient conditions for existence and uniqueness of Bertrand's paradox, mimeo, Penn State and Princeton University.

[3] D. Bernheim (1984), Rationalizable strategic behavior, Econometrica 52, 10071028.

[4] J. Bulow, J. Geanakoplos, and P. Klemperer (1985), Multimarket oligopoly: Strategic substitutes and complements, Journal of Political Economy 93, 488511.

[5] A. Caplin and B. Nalebuff (1991a), Aggregation and social choice: A mean voter theorem, Econometrica 59, 1-23.

[6] A. Caplin and B. Nalebuff (1991b), Aggregation and imperfect competition: On the Existence of Equilibrium, Econometrica 59, 25-59.

[7] R. Cooper and A. John (1988), Coordinating coordination failures in Keynesian models, Quarterly Journal of Economics 103, 441-463.

[8] A. Deaton and J. Muellbauer (1980), "Economics and Consumer Behavior", Cambridge University Press, New York.

[9] E. Dierker (1991), Competition for customers, in "Equilibrium Theory and Applications" (W. Barnett, B. Cornet, C. d'Aspremont, J. Gabszewicz, and A. Mas-Colell, Eds.), Cambridge University Press, Cambridge.

[10] E. Dierker, H. Dierker, and W. Trockel (1984),Price-dispersed preferences and $\mathrm{C}^{1}$ mean demand, Journal of Mathematical Economics 13, 11-42.

[11] B.C. Eaton, B.C. and R.G. Lipsey (1989), Product differentiation, in "Handbook of Industrial Organization" (R. Schmalensee and R.D. Willig, Eds.), vol. 1, pp. 723-763, Amsterdam : North Holland. 
[12] J.-M. Grandmont (1987), Distribution of preferences and the law of demand, Econometrica 55, 155-161.

[13] J.-M. Grandmont (1992), Transformation of the commodity space, behavioral heterogeneity, and the aggregation problem, Journal of Economic Theory 57, $1-35$.

[14] J.-M. Grandmont (1993), Behavioral heterogeneity and Cournot oligopoly equilibrium, Ricerche Economiche 47, 167-187.

[15] P. Klemperer (1995), Competition when consumers have switching costs: An overview with applications to industrial organization, macroeconomics, and international trade", Review of Economic Studies 62, 515-540.

[16] A. Kneip (1993), Heterogeneity of demand behavior and the space of Engel curves, mimeo, University of Bonn.

[17] A. Mas-Colell and W. Neuefeind (1977), Some generic properties of aggregate excess demand and an application, Econometrica 45, 591-599

[18] P. Milgrom and J. Roberts (1990a), The economics of modern manufacturing: Technology, strategy, and organization", American Economic Review 80, 511528.

[19] P. Milgrom and J. Roberts (1990b), Rationalizability, learning and equilibrium in games with strategic complementarities", Econometrica 58, 1255-1277.

[20] P. Milgrom and J. Roberts (1991), Adaptive and sophisticated learning in normal form games, Games and Economic Behavior 3, 82-100.

[21] M. Peitz (1995), Aggregation and strategic complementarity in a model of price competition, University of Bonn discussion paper A-469.

[22] M. Peitz (1997), Differentiated Bertrand duopoly with variable demand, Research in Economics/Ricerche Economiche 51, 85-100.

[23] M. Peitz (1998), Price competition in address models of product differentiation: Unit-elastic demand, mimeo, University of Alicante. 
[24] M. Peitz (1999), A difficulty with the address models of product differentiation, forthcoming in Economic Theory.

[25] A. Prékopa (1973), On logarithmic concave measures and functions, Acta Sci. Math. 34, 335-343.

[26] J. Roberts and H. Sonnenschein (1977), On the foundation of the theory of monopolistic competition, Econometrica 45, 101-113.

[27] T. Tan and S. Werlang (1988), The Bayesian foundations of solution concepts of games, Journal of Economic Theory 45, 370-391.

[28] A. Tversky (1972), Elimination by aspects: A theory of choice, Psychological Review 86, 542-573.

[29] X. Vives (1990), Nash equilibrium with strategic complementarities, Journal of Mathematical Economics 19, 305-321. 